Supporting Information

for

\title{
Palladium-Catalyzed Intramolecular Aminotrifluoromethoxylation of Alkenes
}

\author{
Chaohuang Chen, Pinhong Chen and Guosheng Liu* \\ State Key Laboratory of Organometallic Chemistry, Shanghai Institute of Organic Chemistry, Chinese Academy of \\ Sciences, 345 Lingling Road, Shanghai, China 20032 \\ Email: gliu@mail.sioc.ac.an
}

\section{General Procedures and New Compounds Characterization}

\section{Content :}

\section{General Consideration}

2. Experimental Section

2.1 Synthesis of $\mathrm{CH}_{3} \mathrm{CN}$-solvalated $\mathrm{AgOCF}_{3}$

2.2 Typical procedure for Intramolecular Aminotrifluoromethoxylation of alkenes

2.3 Synthesis and characterization of $\mathbf{2 a - R T s N C O F}$

\section{Mechanism Studies}

3.1 Stereochemistry study

3.2 Procedure for the observation of Aklyl- $\mathrm{OCF}_{3}$ bond elimination from $\mathrm{Pd}(\mathrm{IV})$ center

\subsubsection{Synthesis of Complex 4}

3.2.2. Initial Procedure for the NMR Tracing Experiment.

3.2.3 Synthesis and characterization of compound 9

3.2.4 Synthesis and characterization of compound $\mathbf{1 0}$

\section{New Compounds Characterization}

5. Crystal Structure of $2 \mathrm{f}, 2 \mathrm{r}$ and 10 


\section{General Consideration:}

$\mathrm{Pd}\left(\mathrm{CH}_{3} \mathrm{CN}\right)_{2} \mathrm{Cl}_{2}$ was prepared by refluxing $\mathrm{PdCl}_{2}$ in acetonitrile and other commercial reagents with high purity were purchased and used without further purification, unless otherwise noted. Reactions were monitored by thin-layer chromatography (TLC) carried out on $25 \mathrm{~mm}$ silica gel plates. ${ }^{1} \mathrm{H},{ }^{19} \mathrm{~F}$ and ${ }^{13} \mathrm{C}$ NMR spectra were recorded on an agilent $-400 \mathrm{MHz}$ spectrometer. The chemical shifts $(\delta)$ are given in parts per million relative to internal standard TMS $\left(0 \mathrm{ppm}\right.$ for $\left.{ }^{1} \mathrm{H}\right), \mathrm{CDCl}_{3}$ (77.0 ppm for ${ }^{13} \mathrm{C}$ ). ${ }^{19} \mathrm{~F}$ NMR chemical shifts were determined relative to $\mathrm{CFCl}_{3}$ as outside standard and low field is positive. ${ }^{1} \mathrm{H}$ and ${ }^{19} \mathrm{~F}$ multiplicities are reported as follows: singlet (s), doublet (d), doublet of doublets (dd), quartet (q), multiplet (m), and broad resonance (br). Flash column chromatography was performed on silica gel (particle size 230-400 mesh, purchased from Canada) and eluted with petroleum ether/ethyl acetate. Acetonitrile and THF were directly obtained from solvent purification system of Innovation Technology Company. High Resolution Mass spectral data were obtained on a Waters Micromass GCT spectrometer in EI mode or on Agilent Technologies 6224 TOF LC MS spectrometer in ESI mode.

\section{Experimental Section}

\subsection{Synthesis of $\mathrm{CH}_{3} \mathrm{CN}$-solvalated $\mathrm{AgOCF}_{3}$}

The $\left[\mathrm{AgOCF}_{3}\right]$ stock solution was prepared according to the known procedure reported by Vivic ${ }^{\mathrm{S} 1}$ with slightly modification: In a glove-box, $2.53 \mathrm{~g}$ of $\mathrm{AgF}$ was weighted into a $100 \mathrm{~mL}$ Schlenk flask, and $20 \mathrm{~mL}$ of dry $\mathrm{CH}_{3} \mathrm{CN}$ was then added. The mixture was taken out off the glove-box and cooled in a $\mathrm{CH}_{3} \mathrm{CN} / \mathrm{N}_{2}$ bath (ca. - $40{ }^{\circ} \mathrm{C}$ ). $\mathrm{CF}_{3} \mathrm{SO}_{2} \mathrm{OCF}_{3}{ }^{\mathrm{S} 2}$ was introduced into the reaction mixture by a vacuum transfer, where $\mathrm{CF}_{3} \mathrm{SO}_{2} \mathrm{OCF}_{3}$ was cooled by cold water bath. The mixture was violently stirring in $\mathrm{CH}_{3} \mathrm{CN} / \mathrm{N}_{2}$ bath and warmed to room temperature gradually over-night. After all the $\mathrm{AgF}$ solid was disappeared, the solution was degassed and filtered. The obtained colorless (or light yellow) solution was kept in a sealed tube $(25 \mathrm{~mL})$ in darkness at $-20{ }^{\circ} \mathrm{C}$ for further use. For the result, $\mathrm{AgOCF}_{3}$ solution in $\mathrm{CH}_{3} \mathrm{CN}(\sim 1.0 \mathrm{~mol} / \mathrm{L}, 20 \mathrm{~mL})$ was obtained. ${ }^{19} \mathrm{~F}$ NMR (376 MHz, $\left.\mathrm{CD}_{3} \mathrm{CN}\right): \delta-25.0$ (s, 3F). (lit. $-26.8 \mathrm{ppm}$ ).

2.2 Typical Procedure for Intramolecular Aminotrifluoromethoxylation of alkenes: Alkene substrate $1(0.2 \mathrm{mmol}), \mathrm{Pd}\left(\mathrm{CH}_{3} \mathrm{CN}\right)_{2} \mathrm{Cl}_{2}(5.2 \mathrm{mg}, 0.02 \mathrm{mmol})$, and 
Selectfluor- $\mathrm{BF}_{4}(141.6 \mathrm{mg}, 0.4 \mathrm{mmol})$ were placed into an oven-dried sealed tube (10 $\mathrm{mL})$. After filled with $\mathrm{N}_{2}$ and pre-cooled at $-20{ }^{\circ} \mathrm{C}$, degassed THF $(1.0 \mathrm{~mL})$ was quickly added followed by addition of $\mathrm{CH}_{3} \mathrm{CN}$-solvalated $\mathrm{AgOCF}_{3}(0.6 \mathrm{~mL}, 0.6 \mathrm{mmol}$, $1.0 \mathrm{M}$ ) at same temperature. The mixture was kept in $-20{ }^{\circ} \mathrm{C}$ and stirred for 6 hours. After completion of the reaction, the yellow suspension was diluted by ethyl acetate $(15.0 \mathrm{~mL})$ and filtrated through a short pad of silica gel. The filtrate was concentrated under vacuum, and the crude residue was purified by column chromatography on silica gel with a gradient eluant of petrol ether / ethyl acetate (generally 20:1 15:1) to give desired six-endo products 2 . The results were shown in Table 2.

\subsection{Synthesis and characterization of 2a-RTsNCOF}

Alkene substrate 1a $(0.2 \mathrm{mmol})$ was placed into an oven-dried sealed tube $(10 \mathrm{~mL})$. After filled with $\mathrm{N}_{2}$, degassed $\mathrm{CH}_{3} \mathrm{CN}(1.0 \mathrm{~mL})$ was added followed by addition of $\mathrm{CH}_{3} \mathrm{CN}$-solvalated $\mathrm{AgOCF}_{3}(0.6 \mathrm{~mL}, 0.6 \mathrm{mmol}, 1.0 \mathrm{M})$ at room temperature. The mixture was stirred for 6 hours. After completion of the reaction, the dark suspension was diluted by ethyl acetate $(15.0 \mathrm{~mL})$ and filtrated through a short pad of silica gel. The filtrate was concentrated under vacuum, and the crude residue was purified by column chromatography on silica gel with a gradient eluant of petrol ether / ethyl acetate (20:1 15:1) to give desired product 2a-RTsNCOF in 87\% yield (55 mg).

${ }^{1} \mathrm{H}\left(400 \mathrm{MHz}, \mathrm{CDCl}_{3}\right) \delta 7.82(\mathrm{~d}, J=8.0 \mathrm{~Hz}, 2 \mathrm{H}), 7.34(\mathrm{~d}, J=8.0 \mathrm{~Hz}, 2 \mathrm{H}), 5.90$ $-5.79(\mathrm{~m}, 1 \mathrm{H}), 5.12-5.01(\mathrm{~m}, 2 \mathrm{H}), 3.76(\mathrm{~s}, 2 \mathrm{H}), 2.44(\mathrm{~s}, 3 \mathrm{H}), 2.10(\mathrm{~d}, J=7.6 \mathrm{~Hz}, 2 \mathrm{H})$, $1.02(\mathrm{~s}, 6 \mathrm{H}) .{ }^{19} \mathrm{~F}\left(400 \mathrm{MHz}, \mathrm{CDCl}_{3}\right) \delta 1.33(\mathrm{~s}, 1 \mathrm{~F}) .{ }^{13} \mathrm{C}\left(400 \mathrm{MHz}, \mathrm{CDCl}_{3}\right) \delta 145.90$, $145.14,142.11,134.92$, 133.93, 129.76, 128.68, 118.27, 57.93, 45.14, 35.96, 25.02, 25.01, 21.70. HRMS: m/z (EI) calculated [M]: 313.1148, measured: 313.1147

\section{Mechanism Studies}

\subsection{Stereochemistry Study}

Firstly, we designated the ${ }^{1} \mathrm{H}$ NMR spectrum for product $\mathbf{2 a}$ : 


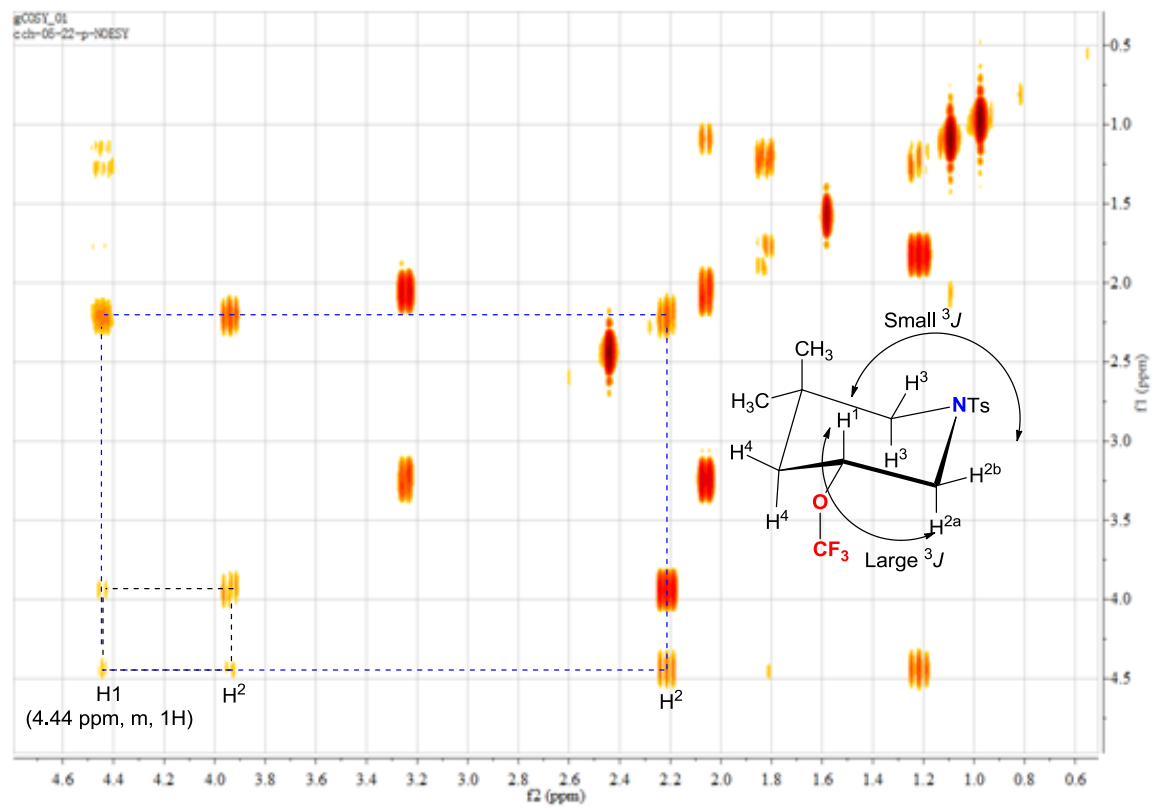

Fig S1. ${ }^{1} \mathrm{H}-{ }^{1} \mathrm{H}$ COESY of product $\mathbf{2 a}$

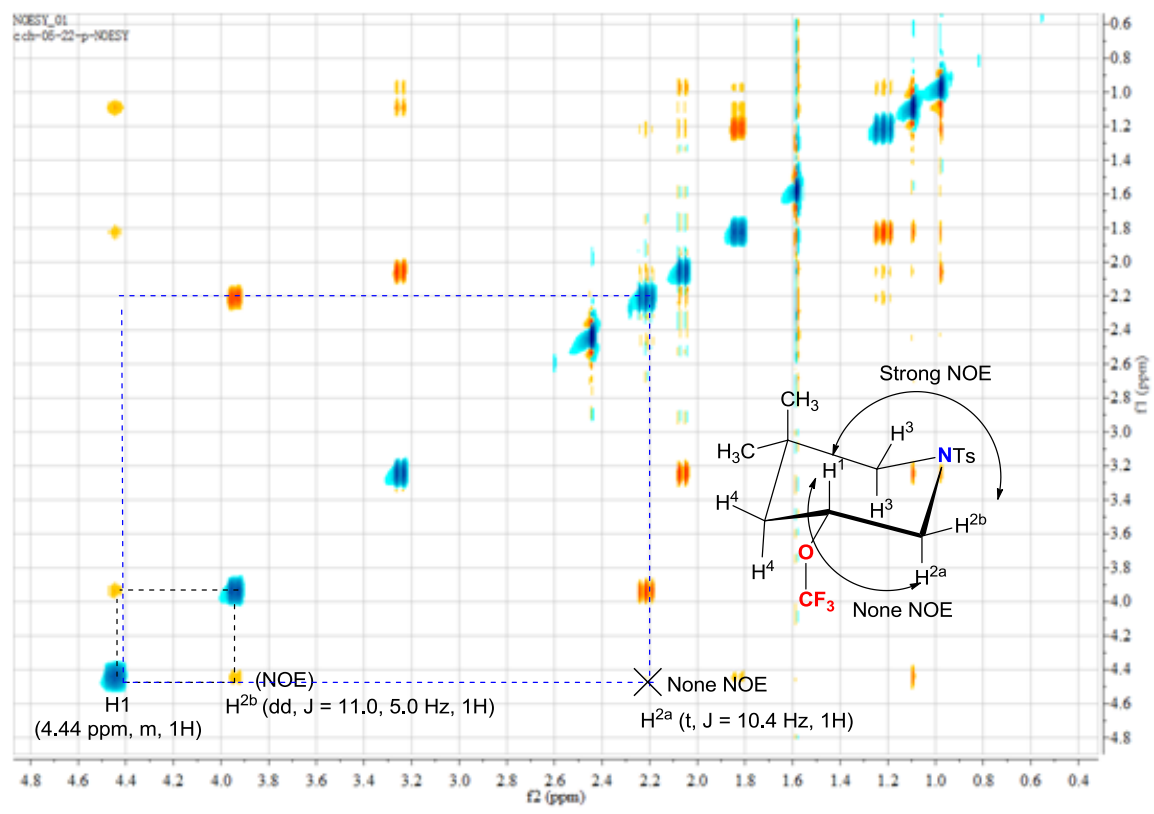

Fig S2. ${ }^{1} \mathrm{H}-{ }^{1} \mathrm{H}$ NOESY of product $\mathbf{2 a}$

According to ${ }^{1} \mathrm{H}-{ }^{1} \mathrm{H}$ COESY and ${ }^{1} \mathrm{H}-{ }^{1} \mathrm{H}$ NOESY of product $\mathbf{2 a}$, we could conclude that the two $\mathrm{H}^{2}$ was at $3.94 \mathrm{ppm} \quad(\mathrm{dd}, J=11.0,5.0 \mathrm{~Hz}, 1 \mathrm{H})$ and $2.21 \mathrm{ppm} \quad(\mathrm{t}, J=10.4$ $\mathrm{Hz}, 1 \mathrm{H})$. According to their $J$-coupling constants and NOE effects with $\mathrm{H}^{1}$ (4.44 ppm, m, $1 \mathrm{H}), 3.94 \mathrm{ppm}$ and $2.21 \mathrm{ppm}$ were designated to be $\mathrm{H}^{2 \mathrm{~b}}$ (cis to $\mathrm{H}^{1}$ ) and $\mathrm{H}^{2 \mathrm{a}}$ (trans to $\mathrm{H}^{1}$ ), respectively. ${ }^{3} \mathrm{H}$ and ${ }^{4} \mathrm{H}$ were similarly designated according to the above analysis. 


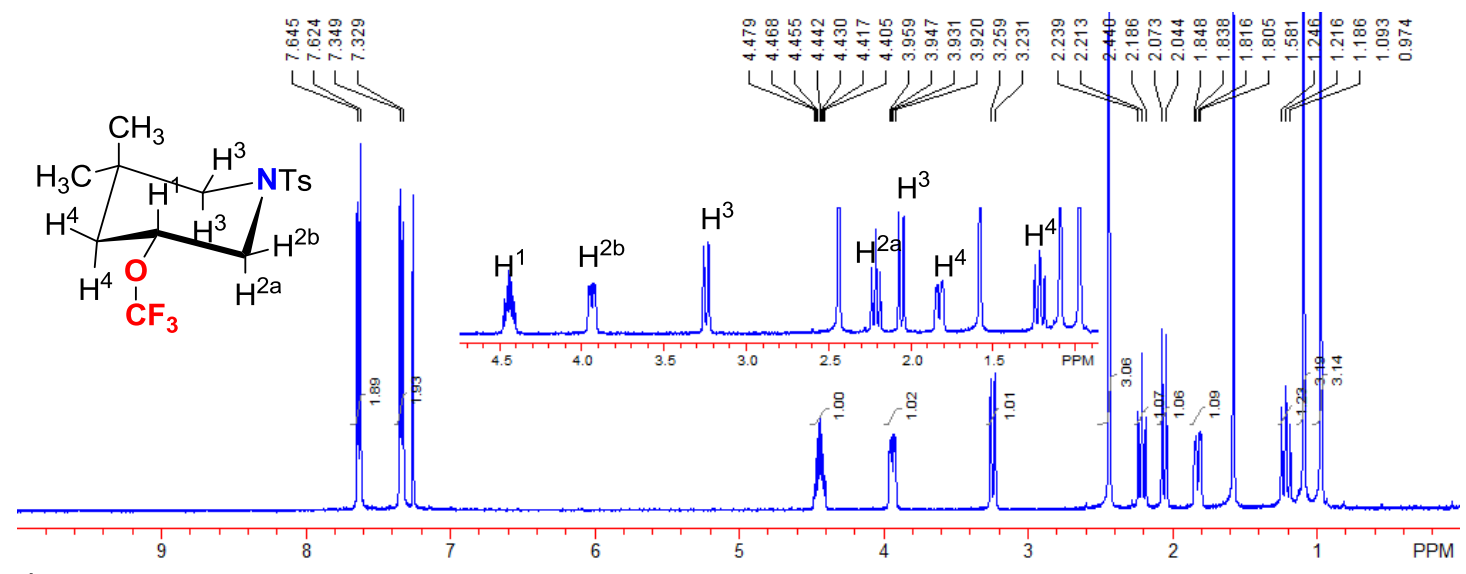

Fig S3. ${ }^{1} \mathrm{H}$ NMR spectrum of product $\mathbf{2 a}$

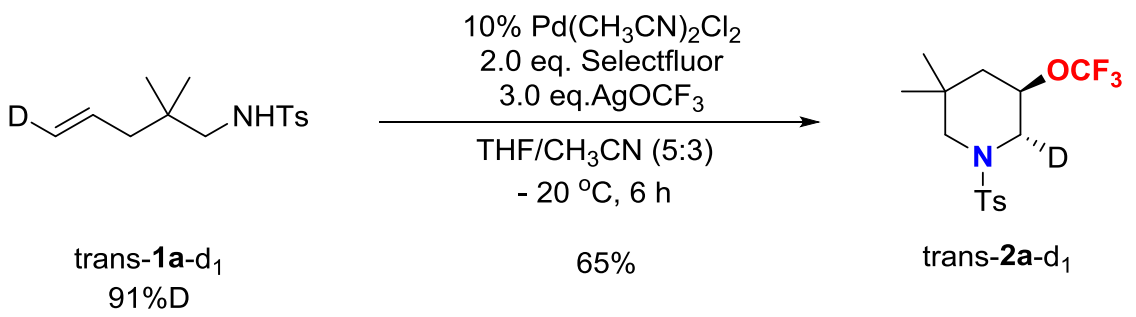

The deuterium-labelled substrate trans-1a- $d_{l}(91 \%$ D containing) was synthesized according to the literature ${ }^{\mathrm{S} 3}$ and then subjected to the reaction conditions. Trans-2a- $d_{1}$ was obtained as a single isomer in $65 \%$ isolated yield (eq. S1). This result indicated that the reaction could involve a trans-aminopalladation followed by direct reductive elimination of a high-valent alkyl-Pd ${ }^{\mathrm{IV}}\left(\mathrm{OCF}_{3}\right)$ complex to yield trans-2a- $d_{1}$.
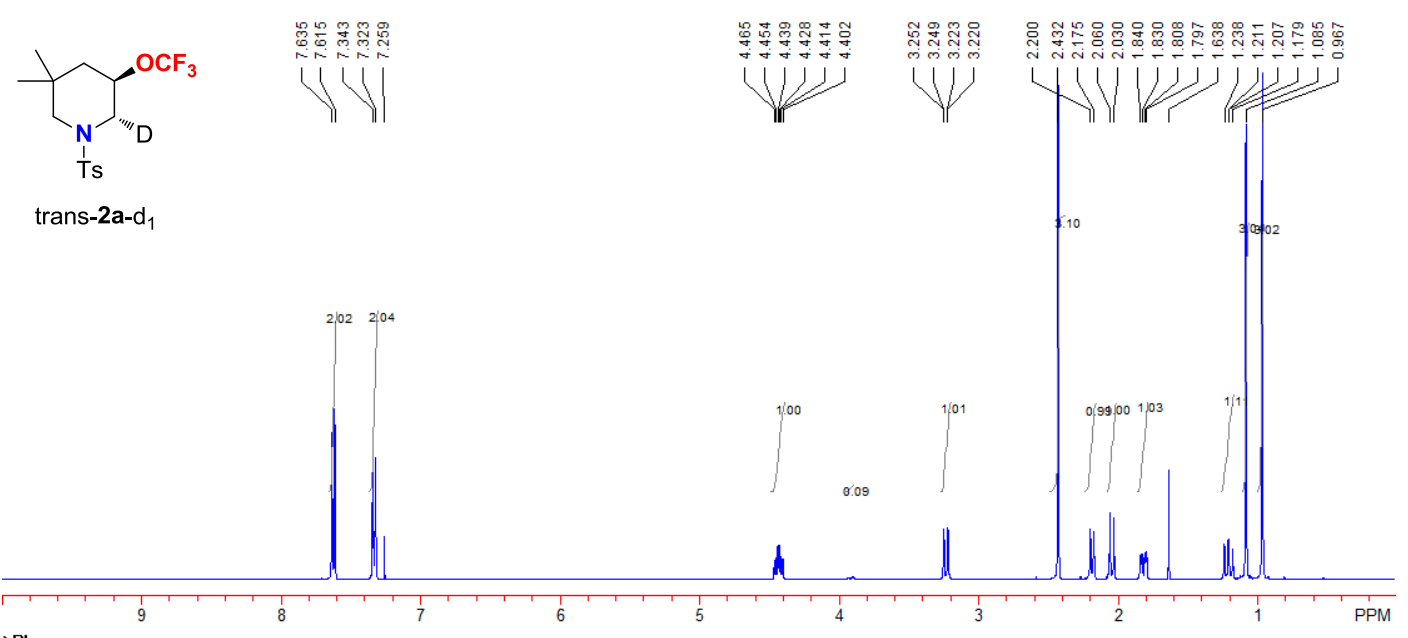

Fig S4. ${ }^{1} \mathrm{H}$ NMR spectrum of trans-2a-d 


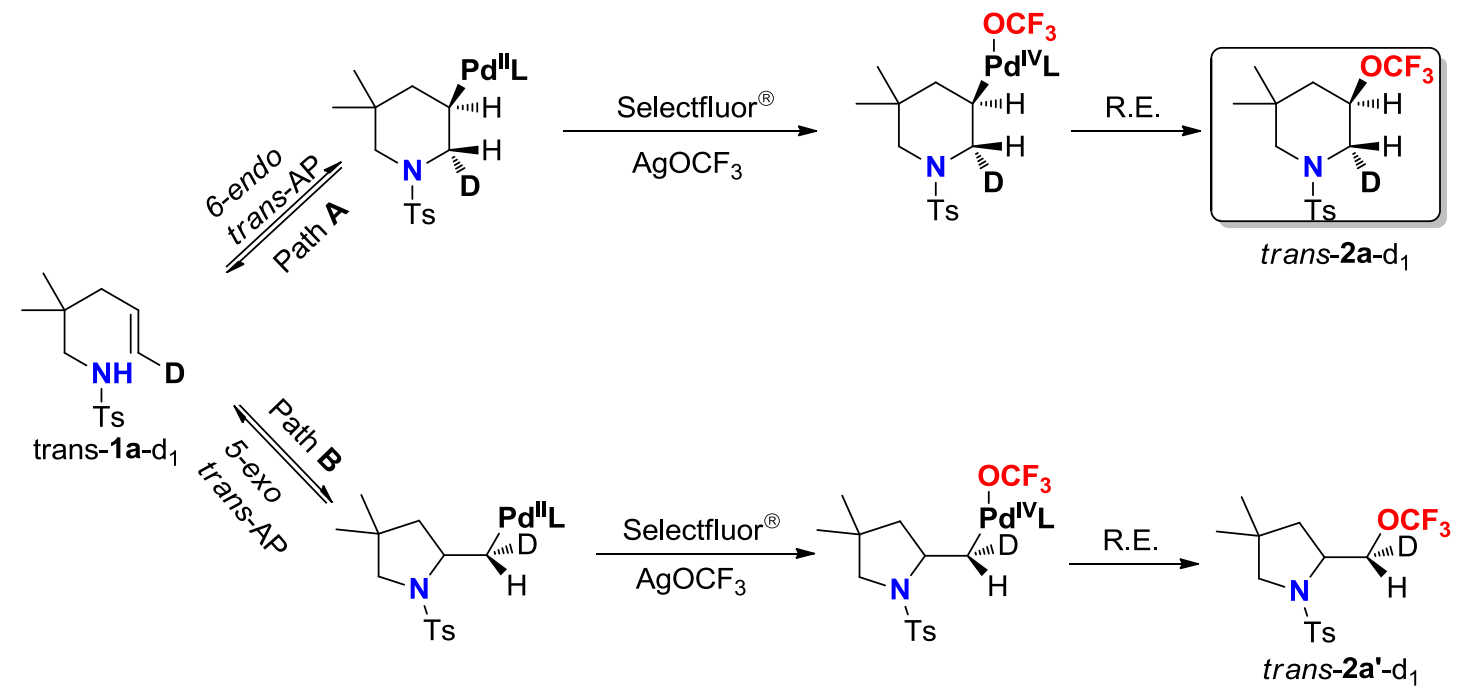

Scheme S1. Proposed mechanism

Based on our previous studies, a possible catalytic cycle is shown in Scheme S1, we proposed Path A is the preferred way: the 6-endo type trans-aminopalladation of alkene generates a secondary $\mathrm{Pd}(\mathrm{II})$ intermediate, which undergoes an oxidation step by selectfluor/AgOCF 3 . The $\mathrm{Pd}(\mathrm{IV})$ intermediate undergoes the direct reductive elimination to form $\mathrm{C}-\mathrm{OCF}_{3}$ bond, where the carbon configuration is retention.<smiles></smiles>

S2

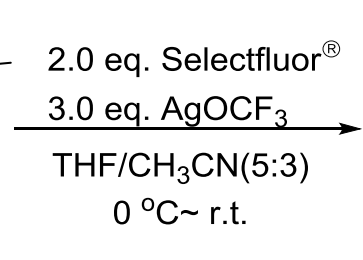

$0{ }^{\circ} \mathrm{C} \sim$ r.t.<smiles>CC1(C)CC(COC(F)(F)F)N(S)CC1(C)COC(F)(F)F</smiles>

2a $55 \%$ 2a-5-exo

$\sim 3 \%$

Again, in order to address the reversible aminopalladation pathway, palladium complex S2 was synthesized ${ }^{\mathrm{S} 4}$ and treated with standard reaction condition (eq S2). Interestingly, 6-membered product $\mathbf{2 a}$ was obtained in 55\% yield, and only trace amount of 5-exo product $(<3 \%)$ was observed. This result strongly demonstrated a reversible aminopalladation of alkene. As a consequence, much more rapidly reductive elimination of secondary alky- $\mathrm{Pd}^{\mathrm{IV}}-\mathrm{OCF}_{3}$ may lead to the formation of 6-endo product, which is consistent with our pervious aminochlorination, aminofluorination, as well as aminooxygenation reactions. 


\subsection{Procedure for Reductive Elimination of $\mathbf{R P d}^{\mathrm{IV}}-\mathrm{OCF}_{3}$ Complex.}

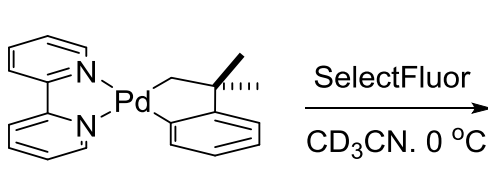

4

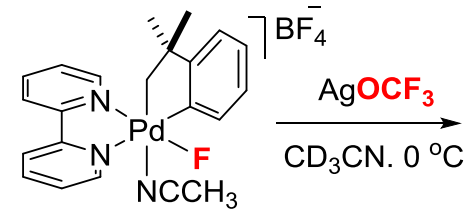

5

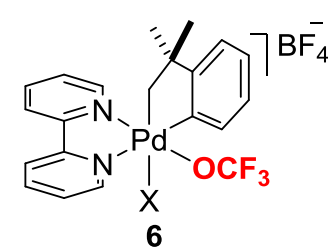

$\mathrm{X}=\mathrm{CH}_{3} \mathrm{CN}$ or $\mathrm{OCF}_{3}$

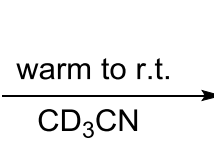

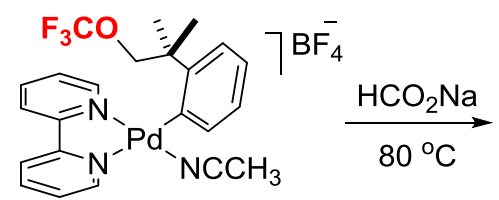

7<smiles>CC(C)(COC(F)(F)F)c1ccccc1</smiles>

11

\subsubsection{Synthesis of Complex 4}

According to the literature, ${ }^{\mathrm{S}} 5$ a complex $\mathrm{Pd}^{\mathrm{II}}\left(\mathrm{CH}_{2} \mathrm{CMe}_{2}-\mathrm{o}-\mathrm{C}_{6} \mathrm{H}_{4}\right)(\mathrm{COD})$ was synthesized. The above palladium complex (150 $\mathrm{mg}, 0.43 \mathrm{mmol})$ was dissolved in $\mathrm{Et}_{2} \mathrm{O}(10 \mathrm{~mL})$ at $-50{ }^{\circ} \mathrm{C}$; then, a solution of 2,2'-bipyridine $(68 \mathrm{mg}, 0.43 \mathrm{mmol})$ in $\mathrm{Et}_{2} \mathrm{O}(5 \mathrm{~mL})$ was added slowly. The reaction mixture was allowed to stir for another 1 $\mathrm{h}$ at room temperature. Precipitates of the solution were collected, and washed with small amount of $\mathrm{Et}_{2} \mathrm{O}$ and hexane, and then dried under vacuum to afford complex 4 as a bright yellow solid (140 mg, 83\% yield). ${ }^{\mathrm{S} 5}{ }^{1} \mathrm{H} \mathrm{NMR}\left(400 \mathrm{MHz}, \mathrm{CDCl}_{3}\right) \delta 9.22(\mathrm{~d}$, $J=4.9 \mathrm{~Hz}, 1 \mathrm{H}), 8.77(\mathrm{~d}, J=4.9 \mathrm{~Hz}, 1 \mathrm{H}), 8.06(\mathrm{dd}, J=8.1,3.0 \mathrm{~Hz}, 2 \mathrm{H}), 7.99-7.91$ $(\mathrm{m}, 2 \mathrm{H}), 7.60-7.51(\mathrm{~m}, 2 \mathrm{H}), 7.48-7.43(\mathrm{~m}, 1 \mathrm{H}), 7.03(\mathrm{dd}, J=5.1,3.7 \mathrm{~Hz}, 2 \mathrm{H}), 6.93$ - $6.88(\mathrm{~m}, 1 \mathrm{H}), 2.48(\mathrm{~s}, 2 \mathrm{H}), 1.46(\mathrm{~s}, 6 \mathrm{H})$. Complex 4 was used directly without further purification.

\subsubsection{Initial Procedure for the NMR Tracing Experiment.}

In a dry-box, compound $4(9.9 \mathrm{mg}, 0.025 \mathrm{mmol})$ and Selectfluor (17.7 $\mathrm{mg}, 0.05$ mmol) were placed into an NMR tube, and then the tube was capped with a rubber plug. The tube was then taken out of the box and was cooled to $0{ }^{\circ} \mathrm{C}$.

$\mathrm{CD}_{3} \mathrm{CN}(0.6 \mathrm{~mL})$ was firstly added at $0{ }^{\circ} \mathrm{C}$, and internal standard $10 \mu \mathrm{L} \mathrm{CF}_{3}$-DMA (1.0 $\mathrm{M}$ in $\mathrm{CDCl}_{3}$ ) was sequentially added. After shacking for 5 seconds, the initial ${ }^{1} \mathrm{H} /{ }^{19} \mathrm{~F}$ NMR spectrum was acquired. Complex 5 was found to form in quantitative yield, which is consistent with literature data. ${ }^{\mathrm{S} 5 \mathrm{~b}}$ 
${ }^{1} \mathrm{H}$ NMR $\left(400 \mathrm{MHz}, \mathrm{CD}_{3} \mathrm{CN}\right) \delta 8.93(\mathrm{~d}, J=5.0 \mathrm{~Hz}, 1 \mathrm{H}), 8.58(\mathrm{~d}, J=8.1 \mathrm{~Hz}, 2 \mathrm{H})$, $8.42(\mathrm{t}, J=7.8 \mathrm{~Hz}, 1 \mathrm{H}), 8.32(\mathrm{t}, J=7.8 \mathrm{~Hz}, 1 \mathrm{H}), 8.01-7.95(\mathrm{~m}, 2 \mathrm{H}), 7.77$ (d, $J=7.8$ $\mathrm{Hz}, 1 \mathrm{H}), 7.59$ (t, $J=6.7 \mathrm{~Hz}, 1 \mathrm{H}), 7.33(\mathrm{t}, J=7.3 \mathrm{~Hz}, 1 \mathrm{H}), 7.26(\mathrm{t}, J=7.1 \mathrm{~Hz}, 1 \mathrm{H})$, $7.11(\mathrm{dd}, J=7.2,1.1 \mathrm{~Hz}, 1 \mathrm{H}), 4.81(\mathrm{dd}, J=10.5,4.9 \mathrm{~Hz}, 1 \mathrm{H}), 4.22(\mathrm{dd}, J=5.0,2.7$ $\mathrm{Hz}, 1 \mathrm{H}), 1.42$ (s, 3H), 1.09 (s, 3H). ${ }^{19} \mathrm{~F}$ NMR (376 MHz, $\left.\mathrm{CD}_{3} \mathrm{CN}\right) \delta-151.41$ (s, 4F), -334.89 (br, s, 1F).

To the above solution of complex 5, $\mathrm{CH}_{3} \mathrm{CN}$-solvated $\mathrm{AgOCF}_{3}(1.0 \mathrm{M}$ in $0.15 \mathrm{~mL})$ was added by syringe, and the mixture was immediately monitored by ${ }^{1} \mathrm{H} /{ }^{19} \mathrm{~F}$ NMR spectrum, respectively. The signals of 5 (at -334.8 ppm for ${ }^{19} \mathrm{~F}-\mathrm{NMR}$, at 4.2 and 4.8 ppm for ${ }^{1} \mathrm{H}-\mathrm{NMR}$ ) were disappeared, and a new broad signal at about $-31.4 \mathrm{ppm}$ $\left({ }^{19} \mathrm{~F}-\mathrm{NMR}\right), 4.0$ and $4.6 \mathrm{ppm}\left({ }^{1} \mathrm{H}-\mathrm{NMR}\right)$ were observed, which possibly belongs the $\mathrm{Pd}^{\mathrm{IV}} \mathrm{OCF}_{3}$ complex 6 in quantitative yield.

${ }^{1} \mathrm{H}$ NMR $\left(400 \mathrm{MHz}, \mathrm{CD}_{3} \mathrm{CN}\right) \delta 8.91(\mathrm{br}, \mathrm{s}, 1 \mathrm{H}), 8.54(\mathrm{~d}, J=7.0 \mathrm{~Hz}, 2 \mathrm{H}), 8.35(\mathrm{t}, J$ $=7.6 \mathrm{~Hz}, 1 \mathrm{H}), 8.26(\mathrm{t}, J=7.6 \mathrm{~Hz}, 1 \mathrm{H}), 8.01-7.82(\mathrm{~m}, 3 \mathrm{H}), 7.51(\mathrm{br}, \mathrm{s}, 1 \mathrm{H}), 7.32-$ $7.16(\mathrm{~m}, 2 \mathrm{H}), 7.06$ (d, $J=7.4 \mathrm{~Hz}, 1 \mathrm{H}), 4.61$ (d, $J=9.4 \mathrm{~Hz}, 1 \mathrm{H}), 4.00$ (br, s, 1H), 1.39 (s, 3H), 1.09 (s, 3H). ${ }^{19} \mathrm{~F}$ NMR (376 MHz, CD 3 CN) $\delta-31.4$ (br, s, 3F), -151.56 (s, 4F).

The above solution in NMR tube was allowed to warm to room temperature (around $25^{\circ} \mathrm{C}$ ). The signal of $\mathbf{6}$ was gradually disappeared, and a series of new signals was appeared, which was assigned to be Pd(II) complex 7.

A 0.05 mmol scale reaction was conducted in a sealed tube according to the above route but with continuous stirring. After completion of the reaction, the solvent was removed in vacuum; the ${ }^{19} F$ yield of compound 7 was calculated to be $43 \%$ with $\mathrm{CF}_{3}$-DMA as internal standard (the yield of compound 8 was about 20\%). ${ }^{1} \mathrm{H} N M R$ $\left(400 \mathrm{MHz}, \mathrm{CD}_{3} \mathrm{CN}\right) \delta 8.71(\mathrm{~d}, \mathrm{~J}=4.8 \mathrm{~Hz}, 1 \mathrm{H}), 8.39(\mathrm{~d}, \mathrm{~J}=8.0 \mathrm{~Hz}, 1 \mathrm{H}), 8.35(d, J=$ $8.0 \mathrm{~Hz}, 1 \mathrm{H}), 8.28(t, J=8.0 \mathrm{~Hz}, 2 \mathrm{H}), 8.16(t, J=9.2 \mathrm{~Hz}, 1 \mathrm{H}), 7.80-7.75(\mathrm{~m}, 2 \mathrm{H})$, $7.40-7.30(\mathrm{~m}, 2 \mathrm{H}), 7.15(t, J=8.0 \mathrm{~Hz}, 1 \mathrm{H}), 7.05(t, J=7.6 \mathrm{~Hz}, 1 \mathrm{H}), 4.50(d, J=9.6$ $H z, 1 H), 4.45(d, J=9.6 \mathrm{~Hz}, 1 \mathrm{H}), 1.73(s, 3 H), 1.69(s, 3 H) .{ }^{19} \mathrm{~F} \mathrm{NMR}(376 \mathrm{MHz}$, $\left.\mathrm{CD}_{3} \mathrm{CN}\right) \delta-60.72(\mathrm{~s}, 3 \mathrm{~F}),-151.66(\mathrm{~s}, 4 \mathrm{~F})$. HRMS: $\mathrm{m} / \mathrm{z}$ (ESI-TOF) calculated $\left[\mathrm{M}-\mathrm{CH}_{3} \mathrm{CN}-\mathrm{BF}_{4}\right]^{+}:$475.0578, measured: 475.0582 . 


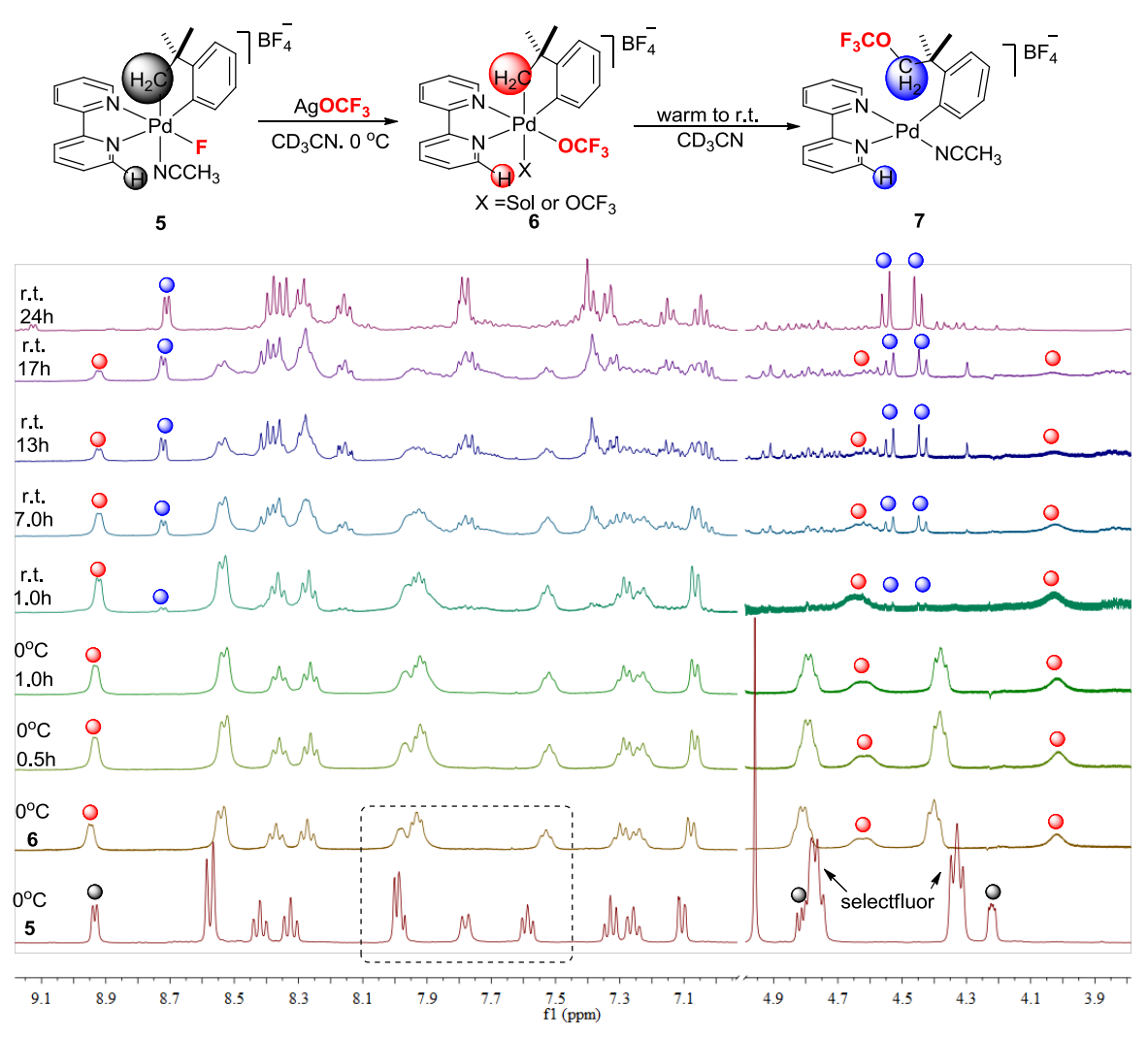

${ }^{1} \mathrm{H}$ MMR of tracing experiment.

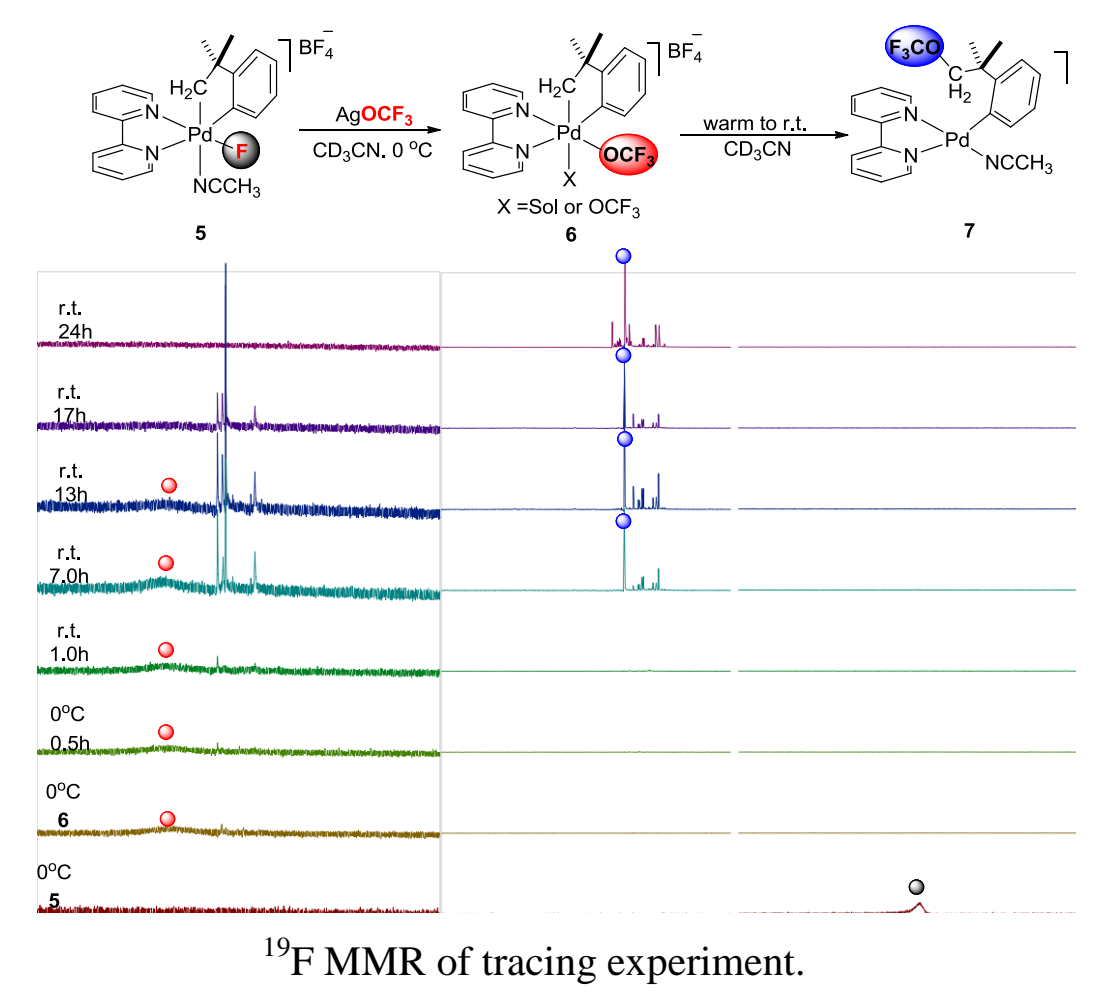

To confirm the structure of 7 , excess amount of $\mathrm{HCO}_{2} \mathrm{Na}$ was added into the solution of complex 7 in $\mathrm{CH}_{3} \mathrm{CN}$, and the mixture was heated to $80{ }^{\circ} \mathrm{C}$ for 2 hours. 
The related hydrogenation product 11 was detected by ${ }^{1} \mathrm{H}$ NMR, ${ }^{19} \mathrm{~F}$ NMR, GC-MS $(\mathrm{m} / \mathrm{z} 218)$. In order to further confirm its structure, compound 11 was also synthesized via an independently procedure (see below). The spectroscopy data of both compounds are consistent.

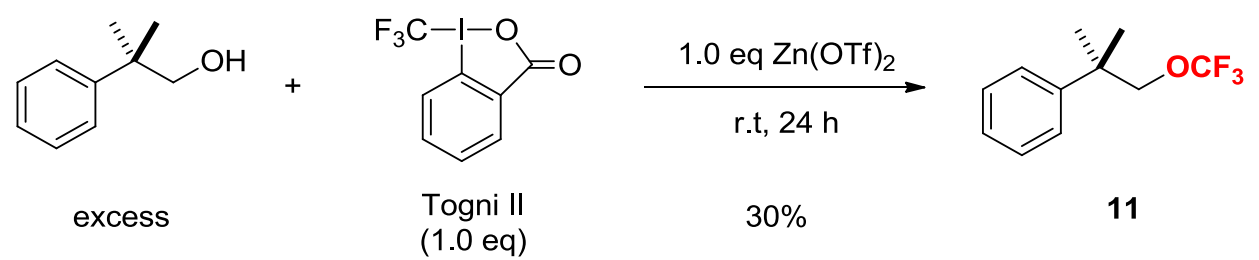

Togni II (63 mg, $0.20 \mathrm{mmol})$ was combined with $\mathrm{Zn}(\mathrm{OTf})_{2}(73 \mathrm{mg}, 0.20 \mathrm{mmol})$ in 2-methyl-2-phenylpropan-1-ol $(1.5 \mathrm{~mL})$. The mixture was stirred for 24 hours at $\mathrm{rt}$. The reaction mixture was purified by flash column chromatography $\left(\mathrm{SiO}_{2}\right.$, pentane/Et $\left.{ }_{2} \mathrm{O}=95: 5\right)$ to yield the title compound as colorless oil in $30 \%$ yield (13 $\mathrm{mg})$.

H NMR (400 MHz, $\left.\mathrm{CDCl}_{3}\right) \delta 7.40-7.30(\mathrm{~m}, 4 \mathrm{H}), 7.27-7.23(\mathrm{~m}, 2 \mathrm{H}), 3.94(\mathrm{~s}, 2 \mathrm{H})$, $1.40(\mathrm{~s}, 6 \mathrm{H}) .{ }^{19} \mathrm{~F}$ NMR $\left(376 \mathrm{MHz}, \mathrm{CDCl}_{3}\right) \delta-60.67$ (s, 3F). ${ }^{13} \mathrm{C}$ NMR (100 MHz, $\left.\mathrm{CDCl}_{3}\right) \delta 145.08,128.39,126.59,125.85,121.69(\mathrm{q}, J=252.4 \mathrm{~Hz}), 75.67(\mathrm{q}, J=2.6$ $\mathrm{Hz}), 38.10$, 30.30, 25.30. HRMS: m/z (EI) calculated [M]: 218.0918, measured: 218.0914 .

In order to get single crystal of Pd(II) complex, pyridine and chloride were added to the solution of complex $\mathbf{7}$ respectively to provide palladium complexes $\mathbf{9}$ and $\mathbf{1 0}$, as shown in eq $\mathbf{S 5}$ and eq $\mathbf{S 6 .}$

\subsubsection{Synthesis and characterization of compound 9}
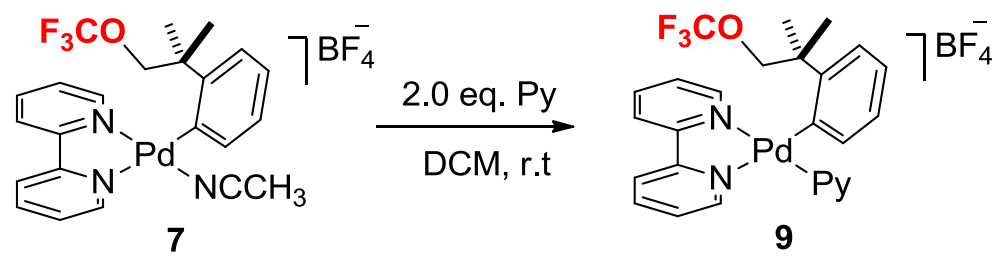

Palladium complex $4(39.8 \mathrm{mg}, 0.1 \mathrm{mmol})$ and Selectfluor $(70.8 \mathrm{mg}, 0.2 \mathrm{mmol})$ were weighted into an oven-dry sealed tube $(10 \mathrm{~mL})$, the tube was vacuumed and refilled with $\mathrm{N}_{2}, 2.0 \mathrm{~mL}$ dry $\mathrm{CH}_{3} \mathrm{CN}$ was then added and the tube was immediately 
cooled to $0{ }^{\circ} \mathrm{C}$. After stirring for 5 minutes, $\mathrm{AgOCF}_{3}(0.6 \mathrm{~mL}, 0.6 \mathrm{mmol}, 1.0 \mathrm{~mol} / \mathrm{L})$ was carefully added in to the reaction mixture, and keep stirring for another 1.0 hours. The reaction was warmed to r.t. and stirring for 24 hours. After completion of the reaction, $\mathrm{CH}_{3} \mathrm{CN}$ solvent was removed by rotary evaporation. The remained crude 7 was dissolved with $10 \mathrm{~mL} \mathrm{CH}_{2} \mathrm{Cl}_{2}$ followed by adding of Pyridine (15.8 mg, 0.2 mmol). The reaction was stirring for 2 hours at room temperature. The mixture was then washed with $3 \times 5 \mathrm{~mL} \mathrm{H}_{2} \mathrm{O}$, dried by $\mathrm{MgSO}_{4}$, then concentrated. The resulting yellow oil was dissolved with $1.0 \mathrm{~mL} \mathrm{CH}_{2} \mathrm{Cl}_{2}$, and precipitated with $10 \mathrm{~mL}$ mixture solution of hexane/ $\mathrm{Et}_{2} \mathrm{O}(\mathrm{v}=1: 1)$. The obtained tacky yellow product was extremely dried under vacuum to give complex 9 as yellow powder $(31 \mathrm{mg}, 39 \%$ yield of $\mathbf{9}$ along with $8 \%$ yield of fluorine-containing Pd(II) complex by ${ }^{19} \mathrm{~F}$ NMR). ${ }^{1} \mathrm{H}$ NMR $\left(400 \mathrm{MHz}, \mathrm{CD}_{2} \mathrm{Cl}_{2}\right) \delta 8.87(\mathrm{~d}, J=5.0 \mathrm{~Hz}, 2 \mathrm{H}), 8.42(\mathrm{~d}, J=8.1 \mathrm{~Hz}, 1 \mathrm{H}), 8.38(\mathrm{~d}, J=$ $8.1 \mathrm{~Hz}, 1 \mathrm{H}), 8.25$ (t, $J=7.2 \mathrm{~Hz}, 1 \mathrm{H}), 8.17(\mathrm{t}, J=7.3 \mathrm{~Hz}, 1 \mathrm{H}), 8.10-8.01(\mathrm{~m}, 2 \mathrm{H})$, $7.73(\mathrm{~d}, J=4.3 \mathrm{~Hz}, 1 \mathrm{H}), 7.68-7.58(\mathrm{~m}, 4 \mathrm{H}), 7.36(\mathrm{t}, J=6.4 \mathrm{~Hz}, 1 \mathrm{H}), 7.26-7.20(\mathrm{~m}$, $1 \mathrm{H}), 7.16-7.08(\mathrm{~m}, 2 \mathrm{H}), 4.20(\mathrm{~d}, J=9.1 \mathrm{~Hz}, 1 \mathrm{H}), 4.01(\mathrm{~d}, J=9.1 \mathrm{~Hz}, 1 \mathrm{H}), 1.71(\mathrm{~s}$, 3H), 1.70 (s, 3H). ${ }^{19} \mathrm{~F}$ NMR (376 MHz, $\left.\mathrm{CD}_{2} \mathrm{Cl}_{2}\right) \delta-60.77$ (s, 3F), -152.70 (s, 4F). ${ }^{13} \mathrm{C}$ NMR (100 MHz, $\left.\mathrm{CD}_{2} \mathrm{Cl}_{2}\right) \delta 156.11,153.82,152.24,152.05,149.99,147.87,147.61$, 140.89 , 140.65, 140.09, 139.81, 133.01, 128.06, 127.62, 127.13, 126.87, 126.77, 126.41, 125.10, 123.50, 123.40, 121.47 (q, $J=254.0 \mathrm{~Hz}), 76.85$ (q, $J=3.8 \mathrm{~Hz}), 39.49$,

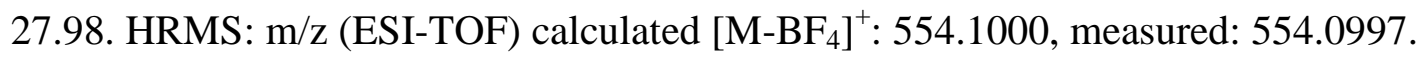

\subsubsection{Synthesis and characterization of compound 10}
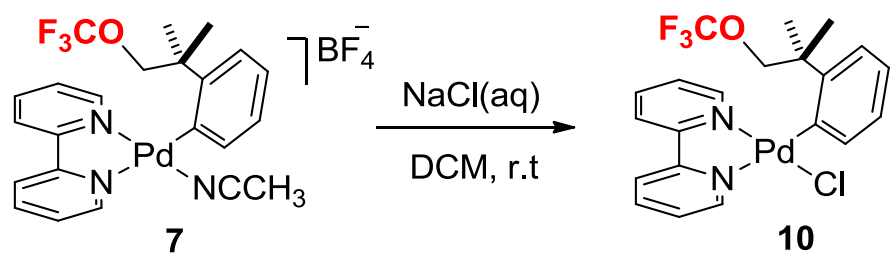

Palladium complex 4 (39.8 mg, $0.1 \mathrm{mmol})$ and Selectfluor (70.8 mg, $0.2 \mathrm{mmol})$ were weighted into an oven-dry sealed tube $(10 \mathrm{~mL})$, the tube was vacuumed and refilled with $\mathrm{N}_{2}, 2.0 \mathrm{~mL}$ dry $\mathrm{CH}_{3} \mathrm{CN}$ was then added and the tube was immediately cooled to $0{ }^{\circ} \mathrm{C}$. After stirring for 5 minutes, $\mathrm{AgOCF}_{3}(0.6 \mathrm{~mL}, 0.6 \mathrm{mmol}, 1.0 \mathrm{~mol} / \mathrm{L})$ was carefully added in to the reaction mixture, and keep stirring for another 1.0 hours. The reaction was warmed to r.t. and stirring for 24 hours. After completion of the reaction, $\mathrm{CH}_{3} \mathrm{CN}$ solvent was removed by rotary evaporation. The remained crude 7 
was dissolved with $10 \mathrm{~mL} \mathrm{CH} \mathrm{Cl}_{2}$ followed by adding of saturated $\mathrm{NaCl}(5.0 \mathrm{~mL})$. The reaction was stirring for 2 hours at room temperature. The mixture was then washed with $3 \times 5 \mathrm{~mL} \mathrm{H}_{2} \mathrm{O}$, dried by $\mathrm{MgSO}_{4}$, then concentrated. The resulting yellow oil was dissolved with $1.0 \mathrm{~mL} \mathrm{CH}_{2} \mathrm{Cl}_{2}$, and precipitated with $10 \mathrm{~mL}$ hexane. The precipates were collected and dried under vacuum to give complex $\mathbf{1 0}$ as light-yellow powder (21 mg, 40\% based on complex 4). Recrystallization of the above product from toluene and hexane afforded X-ray suitable single-crystals of complex $10 .{ }^{1} \mathrm{H}$ NMR (400 MHz, $\left.\mathrm{CDCl}_{3}\right) \delta 9.30(\mathrm{~d}, J=4.4 \mathrm{~Hz}, 1 \mathrm{H}), 8.10-7.98(\mathrm{~m}, 3 \mathrm{H}), 7.94(\mathrm{t}, J=$ $8.0 \mathrm{~Hz}, 1 \mathrm{H}), 7.85(\mathrm{~d}, J=7.2 \mathrm{~Hz}, 1 \mathrm{H}), 7.58(\mathrm{t}, J=6.4 \mathrm{~Hz}, 1 \mathrm{H}), 7.54$ (d, $J=5.6 \mathrm{~Hz}$, 2H), $7.25-7.18(\mathrm{~m}, 2 \mathrm{H}), 6.98(\mathrm{t}, J=7.2 \mathrm{~Hz}, 1 \mathrm{H}), 6.91(\mathrm{t}, \mathrm{J}=7.2 \mathrm{~Hz}, 1 \mathrm{H}), 5.41$ (d, $J$ $=8.8 \mathrm{~Hz}, 1 \mathrm{H}), 4.11(\mathrm{~d}, J=8.8 \mathrm{~Hz}, 1 \mathrm{H}), 1.75(\mathrm{~s}, 3 \mathrm{H}), 1.69(\mathrm{~s}, 3 \mathrm{H}) \cdot{ }^{19} \mathrm{~F}$ NMR $(376$ $\left.\mathrm{MHz}, \mathrm{CDCl}_{3}\right) \delta-60.24$ (s, 3F). ${ }^{13} \mathrm{C} \mathrm{NMR}\left(100 \mathrm{MHz}, \mathrm{CDCl}_{3}\right) \delta 155.64,153.36,151.82$, 149.64, 147.96, 147.44, 139.05, 138.61, 134.80, 127.40, 126.53, 126.41, 124.94, 123.74, 121.83, 121.35, 121.62 (q, $J=252.4 \mathrm{~Hz}), 76.94(\mathrm{q}, J=2.3 \mathrm{~Hz}), 39.60,27.96$, 27.73. HRMS: m/z (ESI-TOF) calculated [M-Cl] $]^{+}$475.0578, measured: 475.0579. 


\section{New Compounds Characterization}

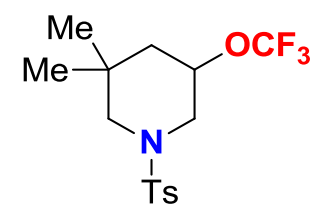

2a

The title compound was obtained as white solid. (50 mg, 72\%). ${ }^{1} \mathrm{H}$ NMR (400 MHz, $\left.\mathrm{CDCl}_{3}\right) \delta 7.63(\mathrm{~d}, J=8.3 \mathrm{~Hz}, 2 \mathrm{H}), 7.34(\mathrm{~d}, J=8.3 \mathrm{~Hz}, 2 \mathrm{H}), 4.44(\mathrm{~m},, 1 \mathrm{H}), 3.94(\mathrm{dd}, J$ $=11.0,5.0 \mathrm{~Hz}, 1 \mathrm{H}), 3.25(\mathrm{~d}, J=11.5 \mathrm{~Hz}, 1 \mathrm{H}), 2.44(\mathrm{~s}, 3 \mathrm{H}), 2.21$ (t, $J=10.4 \mathrm{~Hz}, 1 \mathrm{H})$ $2.06(\mathrm{~d}, J=11.4 \mathrm{~Hz}, 1 \mathrm{H}), 1.83(\mathrm{dd}, J=13.0,4.6 \mathrm{~Hz}, 1 \mathrm{H}), 1.22(\mathrm{dd}, J=12.7,11.1 \mathrm{~Hz}$, 1H), 1.09 (s, 3H), 0.97 (s, 3H). ${ }^{19} \mathrm{~F}$ NMR $\left(376 \mathrm{MHz}, \mathrm{CDCl}_{3}\right) \delta-58.41$ (s, 3F). ${ }^{13} \mathrm{C}$ NMR (100 MHz, $\left.\mathrm{CDCl}_{3}\right) \delta 143.84,133.16,129.81,127.50,121.40$ (q, $J=255.7 \mathrm{~Hz}$ ), 71.19 (q, $J=2.3 \mathrm{~Hz}$ ), 56.45, 49.73, 43.17, 32.04, 28.29, 24.69, 21.54. HRMS: m/z (ESI-TOF) calculated $[\mathrm{M}+\mathrm{H}]^{+}:$352.1189, measured: 352.1192. Anal. Calcd for $\mathrm{C}_{15} \mathrm{H}_{20} \mathrm{~F}_{3} \mathrm{NO}_{3} \mathrm{~S}$ : C, 51.27; H, 5.74; N, 3.99. Found: C, 51.41; H, 5.55; N, 3.66.

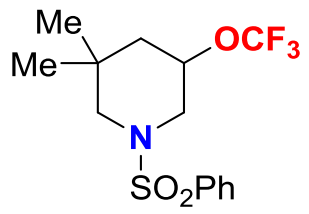

2b

The title compound was obtained as light-yellow oil. (51 mg, 76\%). ${ }^{1} \mathrm{H}$ NMR (400 $\left.\mathrm{MHz}, \mathrm{CDCl}_{3}\right) \delta 7.78-7.73(\mathrm{~m}, 2 \mathrm{H}), 7.65-7.59(\mathrm{~m}, 1 \mathrm{H}), 7.58-7.52(\mathrm{~m}, 2 \mathrm{H}), 4.45$ (m, , 1H), 3.95 (dd, $J=11.1,4.9 \mathrm{~Hz}, 1 \mathrm{H}), 3.26(\mathrm{~d}, J=11.5 \mathrm{~Hz}, 1 \mathrm{H}), 2.25$ (t, $J=10.4$ $\mathrm{Hz}, 1 \mathrm{H}), 2.10(\mathrm{~d}, J=11.5 \mathrm{~Hz}, 1 \mathrm{H}), 1.83(\mathrm{dd}, J=13.1,4.6 \mathrm{~Hz}, 1 \mathrm{H}), 1.23$ (dd, $J=12.7$, $10.9 \mathrm{~Hz}, 1 \mathrm{H}), 1.09$ (s, 3H), 0.98 (s, 3H). ${ }^{19} \mathrm{~F} \mathrm{NMR}\left(376 \mathrm{MHz}, \mathrm{CDCl}_{3}\right) \delta-58.43(\mathrm{~s}, 3 \mathrm{~F})$ ${ }^{13} \mathrm{C} \mathrm{NMR}\left(101 \mathrm{MHz}, \mathrm{CDCl}_{3}\right) \delta 136.26,132.97,129.19,127.40,121.38$ (q, $J=255.7$ $\mathrm{Hz}), 71.11$ (q, $J=2.2 \mathrm{~Hz}), 56.42,49.66,43.11,32.03,28.21,24.68$. HRMS: $\mathrm{m} / \mathrm{z}$ (ESI-TOF) calculated $[\mathrm{M}+\mathrm{H}]^{+}:$338.1032, measured: 338.1036. 


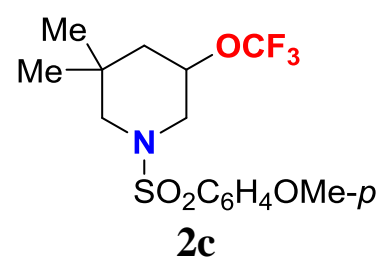

The title compound was obtained as white solid. (58 mg, 78\%). ${ }^{1} \mathrm{H}$ NMR (400 MHz, $\left.\mathrm{CDCl}_{3}\right) \delta 7.69(\mathrm{~d}, J=9.0 \mathrm{~Hz}, 2 \mathrm{H}), 7.00(\mathrm{~d}, J=8.9 \mathrm{~Hz}, 2 \mathrm{H}), 4.44(\mathrm{~m}, 1 \mathrm{H}), 3.92(\mathrm{dd}, J$ $=11.0,4.9 \mathrm{~Hz}, 1 \mathrm{H}), 3.88(\mathrm{~s}, 3 \mathrm{H}), 3.23(\mathrm{~d}, J=11.4 \mathrm{~Hz}, 1 \mathrm{H}), 2.21(\mathrm{t}, J=10.4 \mathrm{~Hz}, 1 \mathrm{H})$, $2.05(\mathrm{~d}, J=11.5 \mathrm{~Hz}, 1 \mathrm{H}), 1.82(\mathrm{dd}, J=13.0,4.5 \mathrm{~Hz}, 1 \mathrm{H}), 1.27-1.15(\mathrm{~m}, 1 \mathrm{H}), 1.09$ (s, $3 \mathrm{H}), 0.97$ (s, 3H). ${ }^{19} \mathrm{~F}$ NMR (376 MHz, $\left.\mathrm{CDCl}_{3}\right) \delta-58.40$ (s, 3F). ${ }^{13} \mathrm{C}$ NMR (101 MHz, $\left.\mathrm{CDCl}_{3}\right) \delta 163.11,129.58,127.70,121.39(\mathrm{q}, J=255.7 \mathrm{~Hz}), 114.33,71.22(\mathrm{q}, J=2.0$ $\mathrm{Hz})$, 56.46, 55.60, 49.73, 43.14, 32.00, 28.28, 24.70. HRMS: m/z (ESI-TOF) calculated $[\mathrm{M}+\mathrm{H}]^{+}: 368.1138$, measured: 368.1142 .

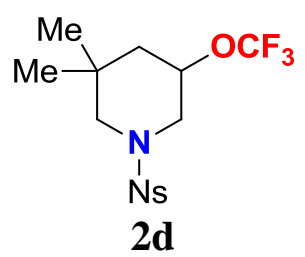

The title compound was obtained as off-white solid. (23 mg, 30\%). ${ }^{1} \mathrm{H}$ NMR (400 $\left.\mathrm{MHz}, \mathrm{CDCl}_{3}\right) \delta 8.39(\mathrm{~d}, J=8.9 \mathrm{~Hz}, 2 \mathrm{H}), 7.95(\mathrm{~d}, J=8.9 \mathrm{~Hz}, 2 \mathrm{H}), 4.45(\mathrm{~m}, 1 \mathrm{H}), 3.94$ $(\mathrm{dd}, J=11.2,4.7 \mathrm{~Hz}, 1 \mathrm{H}), 3.27(\mathrm{~d}, J=11.5 \mathrm{~Hz}, 1 \mathrm{H}), 2.38(\mathrm{t}, J=10.6 \mathrm{~Hz}, 1 \mathrm{H}), 2.22$ (d, $J=11.6 \mathrm{~Hz}, 1 \mathrm{H}), 1.85(\mathrm{dd}, J=13.1,4.1 \mathrm{~Hz}, 1 \mathrm{H}), 1.29(\mathrm{dd}, J=13.0,10.7 \mathrm{~Hz}, 1 \mathrm{H})$, $1.09(\mathrm{~s}, 3 \mathrm{H}), 1.00(\mathrm{~s}, 3 \mathrm{H}) .{ }^{19} \mathrm{~F}$ NMR $\left(376 \mathrm{MHz} \mathrm{CDCl}_{3}\right) \delta-58.53$ (s. 3F). ${ }^{13} \mathrm{C} \mathrm{NMR}$ $\left(101 \mathrm{MHz} \mathrm{CDCl}_{3}\right) \delta 150.25,142.47,128.59,124.52,121.35$ (q, $\left.J=256.0 \mathrm{~Hz}\right), 70.74$ (q, $J=2.4 \mathrm{~Hz}), 56.37,49.48,42.90,32.10,28.03,24.74$. HRMS: m/z (EI) calculated [M]: 382.0810, measured: 382.0802 .

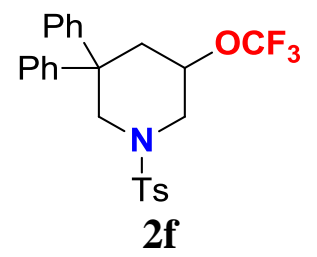

The title compound was obtained as white solid. (76 mg, 80\%). ${ }^{1} \mathrm{H}$ NMR (400 MHz, $\left.\mathrm{CDCl}_{3}\right) \delta 7.65(\mathrm{~d}, J=8.2 \mathrm{~Hz}, 2 \mathrm{H}), 7.49(\mathrm{~d}, J=7.8 \mathrm{~Hz}, 2 \mathrm{H}), 7.38(\mathrm{t}, J=7.8 \mathrm{~Hz}, 2 \mathrm{H})$, 
$7.33(\mathrm{~d}, J=8.1 \mathrm{~Hz}, 2 \mathrm{H}), 7.30-7.16(\mathrm{~m}, 4 \mathrm{H}), 7.12(\mathrm{~d}, J=7.4 \mathrm{~Hz}, 2 \mathrm{H}), 4.62(\mathrm{~d}, J=$ $12.3 \mathrm{~Hz}, 1 \mathrm{H}), 4.22(\mathrm{~m}, 1 \mathrm{H}), 4.07$ (dd, $J=10.3,4.9 \mathrm{~Hz}, 1 \mathrm{H}), 2.99$ (d, $J=12.5 \mathrm{~Hz}, 1 \mathrm{H})$, $2.43(\mathrm{~s}, 3 \mathrm{H}), 2.32$ (d, $J=12.4 \mathrm{~Hz}, 1 \mathrm{H}), 2.24(\mathrm{t}, J=10.3 \mathrm{~Hz}, 1 \mathrm{H}), 2.14(\mathrm{t}, J=12.0 \mathrm{~Hz}$, 1H). ${ }^{19} \mathrm{~F}$ NMR $\left(376 \mathrm{MHz}, \mathrm{CDCl}_{3}\right) \delta-58.45$ (s, 3F). ${ }^{13} \mathrm{C} \mathrm{NMR}\left(100 \mathrm{MHz}, \mathrm{CDCl}_{3}\right) \delta$ $145.15,144.20,142.35,131.99,129.96,128.89$, 128.67, 127.73, 127.63, 127.00, 126.71, 126.28, 121.35 (q, $J=256.3 \mathrm{~Hz}), 71.02$ (q, $J=2.3 \mathrm{~Hz}), 53.74,49.77,46.36$, 41.08, 21.56. HRMS: m/z (ESI-TOF) calculated $\left[\mathrm{M}+\mathrm{NH}_{4}\right]^{+}:$493.1767, measured: 493.1770. Anal. Calcd for $\mathrm{C}_{25} \mathrm{H}_{24} \mathrm{~F}_{3} \mathrm{NO}_{3} \mathrm{~S}$ : C, 63.14; H, 5.09; N, 2.95. Found: C, 63.03; H, 5.09; N, 2.95.

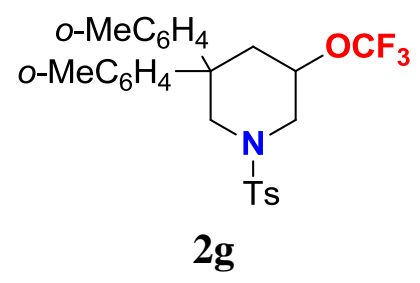

The title compound was obtained as light-yellow oil. (60 mg, 60\%). ${ }^{1} \mathrm{H}$ NMR (400 MHz, $\left.\mathrm{CDCl}_{3}\right) \delta 8.16(\mathrm{~d}, J=6.6 \mathrm{~Hz}, 1 \mathrm{H}), 7.63(\mathrm{~d}, J=8.0 \mathrm{~Hz}, 2 \mathrm{H}), 7.43(\mathrm{~d}, J=7.5 \mathrm{~Hz}, 1 \mathrm{H})$, $7.37-7.25$ (m, 3H), 7.19 (dt, $J=14.4,7.2 \mathrm{~Hz}, 3 \mathrm{H}), 7.03$ (t, $J=6.9 \mathrm{~Hz}, 2 \mathrm{H}), 4.77$ (d, $J=12.0 \mathrm{~Hz}, 1 \mathrm{H}), 4.25-4.07(\mathrm{~m}, 2 \mathrm{H}), 2.92(\mathrm{~d}, J=11.8 \mathrm{~Hz}, 1 \mathrm{H}), 2.43(\mathrm{~s}, 3 \mathrm{H}), 2.33-$ $2.15(\mathrm{~m}, 3 \mathrm{H}), 1.79$ (s, 3H), 1.72 (s, 3H). ${ }^{19} \mathrm{~F}$ NMR (376 MHz, $\left.\mathrm{CDCl}_{3}\right) \delta-58.42$ (s, 3F). ${ }^{13} \mathrm{C}$ NMR $\left(100 \mathrm{MHz}, \mathrm{CDCl}_{3}\right) \delta 144.17,141.90,139.93,137.04,135.88,133.58$, 133.09, 131.91, 130.54, 129.97, 127.74, 127.40, 127.10, 126.26, 125.99, 125.92, $121.28(\mathrm{q}, J=256.4 \mathrm{~Hz}), 71.56(\mathrm{q}, J=2.2 \mathrm{~Hz}), 52.87,49.71,47.13,39.96,22.03$, 21.55, 21.26. HRMS: m/z (EI) calculated [M]: 503.1742, measured: 503.1747.

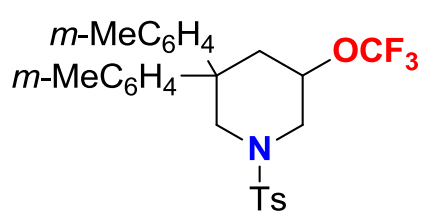

$2 h$

The title compound was obtained as white solid. ( $32 \mathrm{mg}, 63 \%, 0.1 \mathrm{mmol} \mathrm{scale}$ ). ${ }^{1} \mathrm{H}$ $\operatorname{NMR}\left(400 \mathrm{MHz}, \mathrm{CDCl}_{3}\right) \delta 7.66(\mathrm{~d}, J=8.1 \mathrm{~Hz}, 2 \mathrm{H}), 7.37-7.23(\mathrm{~m}, 5 \mathrm{H}), 7.16(\mathrm{t}, J=$ $7.6 \mathrm{~Hz}, 1 \mathrm{H}), 7.06(\mathrm{~d}, J=6.9 \mathrm{~Hz}, 1 \mathrm{H}), 7.01(\mathrm{~d}, J=7.5 \mathrm{~Hz}, 1 \mathrm{H}), 6.90-6.98(\mathrm{~m}, 2 \mathrm{H})$, 
$4.63(\mathrm{~d}, J=12.3 \mathrm{~Hz}, 1 \mathrm{H}), 4.18-4.31(\mathrm{~m}, 1 \mathrm{H}), 4.07$ (dd, $J=10.2,4.8 \mathrm{~Hz}, 1 \mathrm{H}), 2.99$ (d, $J=12.4 \mathrm{~Hz}, 1 \mathrm{H}), 2.43(\mathrm{~s}, 3 \mathrm{H}), 2.37(\mathrm{~s}, 3 \mathrm{H}), 2.30-2.27(\mathrm{~m}, 1 \mathrm{H}), 2.29(\mathrm{~s}, 3 \mathrm{H}), 2.24(\mathrm{t}$, $J=10.4 \mathrm{~Hz}, 1 \mathrm{H}), 2.11(\mathrm{t}, J=12.0 \mathrm{~Hz}, 1 \mathrm{H}) .{ }^{19} \mathrm{~F} \mathrm{NMR}\left(376 \mathrm{MHz}, \mathrm{CDCl}_{3}\right) \delta-58.39(\mathrm{~s}$, 3F). ${ }^{13} \mathrm{C}$ NMR (100 MHz, $\left.\mathrm{CDCl}_{3}\right) \delta 145.19,144.11,142.26,138.32,138.25,132.18$, $129.94,128.69,128.50,128.29,127.71,127.39,126.85,124.63,123.34,121.39$ (q, $J$ $=256.2 \mathrm{~Hz}), 71.15(\mathrm{q}, J=2.3 \mathrm{~Hz}), 53.79,49.78,46.13,41.09,21.72,21.62,21.54$. HRMS: m/z (ESI-TOF) calculated $\left[\mathrm{M}+\mathrm{NH}_{4}\right]^{+}:$521.2080, measured: 521.2084.

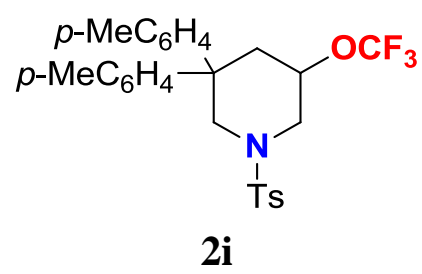

The title compound was obtained as white solid. (70 mg, 70\%). ${ }^{1} \mathrm{H}$ NMR (400 MHz, $\left.\mathrm{CDCl}_{3}\right) \delta 7.64(\mathrm{~d}, J=8.2 \mathrm{~Hz}, 2 \mathrm{H}), 7.36(\mathrm{~d}, J=8.2 \mathrm{~Hz}, 2 \mathrm{H}), 7.33(\mathrm{~d}, J=8.0 \mathrm{~Hz}, 2 \mathrm{H})$, $7.17(\mathrm{~d}, J=8.0 \mathrm{~Hz}, 2 \mathrm{H}), 7.07(\mathrm{~d}, J=8.2 \mathrm{~Hz}, 2 \mathrm{H}), 7.00(\mathrm{~d}, J=8.3 \mathrm{~Hz}, 2 \mathrm{H}), 4.58(\mathrm{~d}, J$ $=12.3 \mathrm{~Hz}, 1 \mathrm{H}), 4.28-4.18(\mathrm{~m}, 1 \mathrm{H}), 4.06(\mathrm{dd}, J=10.2,5.0 \mathrm{~Hz}, 1 \mathrm{H}), 2.95(\mathrm{~d}, J=12.4$ $\mathrm{Hz}, 1 \mathrm{H}), 2.43$ (s, 3H), 2.34 (s, 3H), $2.30-2.25$ (m, 1H), 2.28 (s, 3H), 2.21 (t, $J=10.3$ $\mathrm{Hz}, 1 \mathrm{H}), 2.09$ (t, $J=12.0 \mathrm{~Hz}, 1 \mathrm{H}) .{ }^{19} \mathrm{~F}$ NMR $\left(376 \mathrm{MHz}, \mathrm{CDCl}_{3}\right) \delta-58.40(\mathrm{~s}, 3 \mathrm{~F}) .{ }^{13} \mathrm{C}$ NMR (100 MHz, $\left.\mathrm{CDCl}_{3}\right) \delta 144.10,142.51,139.39$, 136.62, 136.16, 132.08, 129.91, 129.57, 129.29, 127.72, 127.40, 126.10, 121.37 (q, $J=256.2 \mathrm{~Hz}), 71.15$ (q, $J=2.2$ $\mathrm{Hz}$ ) 53.88, 49.77, 45.77, 41.08, 21.54, 20.94, 20.84. HRMS: m/z (ESI-TOF) calculated $\left[\mathrm{M}+\mathrm{NH}_{4}\right]^{+}:$521.2080, measured: 521.2083.

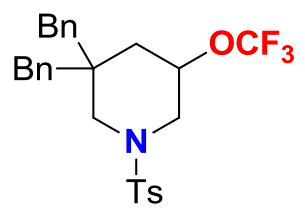

$2 \mathbf{j}$

The title compound was obtained as white crystal. (71 mg, 71\%). ${ }^{1} \mathrm{H}$ NMR (400 $\left.\mathrm{MHz}, \mathrm{CDCl}_{3}\right) \delta 7.62(\mathrm{~d}, J=8.1 \mathrm{~Hz}, 2 \mathrm{H}), 7.41-7.27(\mathrm{~m}, 7 \mathrm{H}), 7.26-7.16(\mathrm{~m}, 3 \mathrm{H})$, $6.95(\mathrm{~d}, J=6.8 \mathrm{~Hz}, 2 \mathrm{H}), 4.62-4.76(\mathrm{~m}, 1 \mathrm{H}), 3.90(\mathrm{dd}, J=10.4,4.4 \mathrm{~Hz}, 1 \mathrm{H}), 3.48$ (d, $J$ $=11.4 \mathrm{~Hz}, 1 \mathrm{H}), 2.99(\mathrm{~d}, J=13.7 \mathrm{~Hz}, 1 \mathrm{H}), 2.87(\mathrm{~d}, J=13.7 \mathrm{~Hz}, 1 \mathrm{H}), 2.64(\mathrm{~d}, J=13.7$ $\mathrm{Hz}, 1 \mathrm{H}), 2.56(\mathrm{~d}, J=13.7 \mathrm{~Hz}, 1 \mathrm{H}), 2.43(\mathrm{~s}, 3 \mathrm{H}), 2.12$ (d, $J=11.5 \mathrm{~Hz}, 1 \mathrm{H}), 1.94$ (t, $J=$ $10.3 \mathrm{~Hz}, 1 \mathrm{H}), 1.86(\mathrm{dd}, J=13.0,3.7 \mathrm{~Hz}, 1 \mathrm{H}), 1.32-1.19(\mathrm{~m}, 1 \mathrm{H}) .{ }^{19} \mathrm{~F}$ NMR $(376$ 
$\left.\mathrm{MHz}, \mathrm{CDCl}_{3}\right) \delta-58.39$ (s, 3F). ${ }^{13} \mathrm{C} \mathrm{NMR}\left(101 \mathrm{MHz}, \mathrm{CDCl}_{3}\right) \delta 144.10,136.65,136.03$, $132.31,130.89,130.87,129.77,128.35,128.10,127.64,126.79,126.62,121.43$ (q, $J$ $=255.9 \mathrm{~Hz}), 71.10(\mathrm{q}, J=2.2 \mathrm{~Hz}), 52.10,49.69,44.04,42.22,38.85,37.34,21.54$. HRMS: m/z (EI) calculated [M] $]^{+}: 503.1735$, measured: 503.1737.

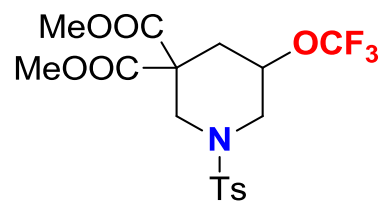

$2 \mathrm{k}$

The title compound was obtained as light-yellow oil. (32 mg, 37\%). ${ }^{1} \mathrm{H}$ NMR (400 $\left.\mathrm{MHz}, \mathrm{CDCl}_{3}\right) \delta 7.65(\mathrm{~d}, J=8.1 \mathrm{~Hz}, 2 \mathrm{H}), 7.35(\mathrm{~d}, J=7.9 \mathrm{~Hz}, 2 \mathrm{H}), 4.61-4.76(\mathrm{~m}, 1 \mathrm{H})$, $4.24(\mathrm{~d}, J=12.2 \mathrm{~Hz}, 1 \mathrm{H}), 3.96$ (dd, $J=11.0,5.1 \mathrm{~Hz}, 1 \mathrm{H}), 3.80$ (s, 3H), 3.73 (s, 3H), $2.81(\mathrm{dd}, J=12.9,4.2 \mathrm{~Hz}, 1 \mathrm{H}), 2.68(\mathrm{~d}, J=12.3 \mathrm{~Hz}, 1 \mathrm{H}), 2.44$ (s, 3H), 2.27 (t, $J=$ $10.6 \mathrm{~Hz}, 1 \mathrm{H}), 1.58(\mathrm{dd}, J=13.0,10.7 \mathrm{~Hz}, 1 \mathrm{H}) .{ }^{19} \mathrm{~F}$ NMR $\left(376 \mathrm{MHz}, \mathrm{CDCl}_{3}\right) \delta-58.79$ (s, 3F). ${ }^{13} \mathrm{C}$ NMR $\left(100 \mathrm{MHz}, \mathrm{CDCl}_{3}\right) \delta 168.57,168.31,144.35,132.77,130.01$, 127.59, 121.31 (q, $J=257.0 \mathrm{~Hz}), 69.63(\mathrm{q}, J=2.4 \mathrm{~Hz}), 54.09,53.44,53.27,49.53$, 49.36, 35.01, 21.55. HRMS: m/z (EI) calculated [M]: 439.0913, measured: 439.0906.

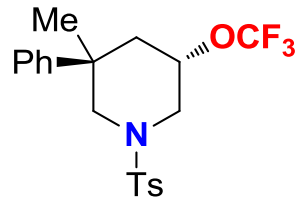

trans-2l

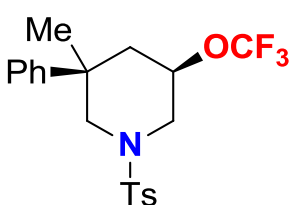

cis-2l

The title compound was obtained as white solid. $(50 \mathrm{mg}, 60 \%$ yield, trans: $c$ is $=$ 3.4:1). trans-2I: ${ }^{1} \mathrm{H}$ NMR (400 MHz, $\left.\mathrm{CDCl}_{3}\right) \delta 7.64(\mathrm{~d}, J=8.2 \mathrm{~Hz}, 2 \mathrm{H}), 7.43-7.20$ $(\mathrm{m}, 7 \mathrm{H}), 4.74-4.56(\mathrm{~m}, 1 \mathrm{H}), 4.16(\mathrm{dd}, J=10.8,5.3 \mathrm{~Hz}, 1 \mathrm{H}), 3.80(\mathrm{~d}, J=11.5 \mathrm{~Hz}$, $1 \mathrm{H}), 2.42(\mathrm{~s}, 3 \mathrm{H}), 2.32(\mathrm{dd}, J=12.5,6.4 \mathrm{~Hz}, 1 \mathrm{H}), 2.19(\mathrm{t}, J=10.7 \mathrm{~Hz}, 1 \mathrm{H}), 1.73(\mathrm{t}, J$ $=12.1 \mathrm{~Hz}, 1 \mathrm{H}), 1.49(\mathrm{~s}, 3 \mathrm{H}) .{ }^{19} \mathrm{~F}$ NMR $\left(376 \mathrm{MHz}, \mathrm{CDCl}_{3}\right) \delta-58.38(\mathrm{~s}, 3 \mathrm{~F}) .{ }^{13} \mathrm{C} \mathrm{NMR}$ $\left(100 \mathrm{MHz}, \mathrm{CDCl}_{3}\right) \delta 145.58,144.00,133.05,129.92,128.70,127.46,127.03,124.94$, $121.43(\mathrm{q}, J=256.0 \mathrm{~Hz}), 71.32(\mathrm{q}, J=1.7 \mathrm{~Hz}), 55.42,49.82,41.48,38.72,24.66$, 21.54. cis-2I: ${ }^{1} \mathrm{H}$ NMR (400 MHz, $\left.\mathrm{CDCl}_{3}\right) \delta 7.67(\mathrm{~d}, J=8.2 \mathrm{~Hz}, 2 \mathrm{H}), 7.45(\mathrm{~d}, J=8.1$ $\mathrm{Hz}, 2 \mathrm{H}), 7.38$ (t, $J=8.7 \mathrm{~Hz}, 4 \mathrm{H}), 7.28-7.22(\mathrm{~m}, 1 \mathrm{H}), 4.22(\mathrm{~d}, J=12.0 \mathrm{~Hz}, 1 \mathrm{H}), 4.19$ $-4.10(\mathrm{~m}, 1 \mathrm{H}), 3.87(\mathrm{dd}, J=10.6,4.8 \mathrm{~Hz}, 1 \mathrm{H}), 2.66(\mathrm{~d}, J=13.3 \mathrm{~Hz}, 1 \mathrm{H}), 2.46(\mathrm{~s}, 3 \mathrm{H})$, 
2.22-2.31 (m, 2H), $1.49(\mathrm{dd}, J=13.2,11.2 \mathrm{~Hz}, 1 \mathrm{H}), 1.22(\mathrm{~s}, 3 \mathrm{H}) .{ }^{19} \mathrm{~F}$ NMR $(376 \mathrm{MHz}$, $\left.\mathrm{CDCl}_{3}\right) \delta-58.47(\mathrm{~s}, 3 \mathrm{~F}) .{ }^{13} \mathrm{C} \mathrm{NMR}\left(100 \mathrm{MHz}, \mathrm{CDCl}_{3}\right) \delta$ 144.07, 143.32, 132.27, 129.87, 128.90, 127.74, 126.58, 125.81, 121.32 (q, $J=256.0 \mathrm{~Hz}), 70.64(\mathrm{~d}, J=2.2$ $\mathrm{Hz})$, 54.01, 49.75, 42.71, 38.98, 30.58, 21.56. HRMS: m/z (EI) calculated [M]: 413.1273, measured: 413.1268 .

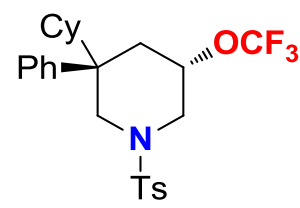

trans-2m

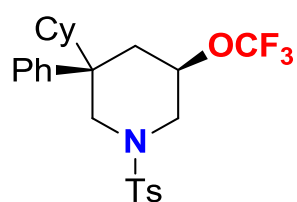

cis-2m

The title compound was obtained as white solid $(73 \mathrm{mg}, 76 \%$ yield, trans: $\mathrm{cis}=$ 1.1:1). trans-2m: ${ }^{1} \mathrm{H} \mathrm{NMR}\left(400 \mathrm{MHz}, \mathrm{CDCl}_{3}\right) \delta 7.70(\mathrm{~d}, J=8.2 \mathrm{~Hz}, 2 \mathrm{H}), 7.41-7.21$ (m, 5H), $7.15(\mathrm{~d}, J=7.5 \mathrm{~Hz}, 2 \mathrm{H}), 4.65-4.55(\mathrm{~m}, 1 \mathrm{H}), 4.46(\mathrm{t}, J=12.7 \mathrm{~Hz}, 1 \mathrm{H}), 4.12$ (dd, $J=10.4,5.1 \mathrm{~Hz}, 1 \mathrm{H}), 2.95(\mathrm{~d}, J=13.2 \mathrm{~Hz}, 1 \mathrm{H}), 2.42(\mathrm{~s}, 3 \mathrm{H}), 2.23-2.17(\mathrm{~m}, 2 \mathrm{H})$, $1.99-0.60(\mathrm{~m}, 11 \mathrm{H}), 0.58-0.43(\mathrm{~m}, 1 \mathrm{H}) .{ }^{19} \mathrm{~F} \mathrm{NMR}\left(376 \mathrm{MHz}, \mathrm{CDCl}_{3}\right) \delta-58.43(\mathrm{~s}$, 3F). ${ }^{13} \mathrm{C}$ NMR $\left(100 \mathrm{MHz}, \mathrm{CDCl}_{3}\right) \delta 144.02,141.49,132.85,129.92,128.35,127.80$, 127.53, 126.53, $121.46(\mathrm{~d}, J=256.0 \mathrm{~Hz}), 70.82(\mathrm{~d}, J=2.2 \mathrm{~Hz}), 51.52,49.89,47.98$, 44.51, 39.39, 27.36, 26.83, 26.80, 26.57, 26.02, 21.52. cis-2m: ${ }^{1} \mathrm{H}$ NMR (400 MHz, $\left.\mathrm{CDCl}_{3}\right) \delta 7.65(\mathrm{~d}, J=8.2 \mathrm{~Hz}, 2 \mathrm{H}), 7.41-7.32(\mathrm{~m}, 5 \mathrm{H}), 7.30(\mathrm{~d}, J=7.7 \mathrm{~Hz}, 2 \mathrm{H}), 4.46$ (t, $J=12.7 \mathrm{~Hz}, 1 \mathrm{H}), 4.23-4.15(\mathrm{~m}, 1 \mathrm{H}), 3.87(\mathrm{dd}, J=10.2,5.3 \mathrm{~Hz}, 1 \mathrm{H}), 2.82(\mathrm{~d}, J=$ $13.2 \mathrm{~Hz}, 1 \mathrm{H}), 2.46(\mathrm{~s}, 3 \mathrm{H}), 2.17-2.10(\mathrm{~m}, 2 \mathrm{H}), 1.96-0.59(\mathrm{~m}, 12 \mathrm{H}) .{ }^{19} \mathrm{~F}$ NMR $(376$ $\left.\mathrm{MHz}, \mathrm{CDCl}_{3}\right) \delta-58.42$ (s, 3F). ${ }^{13} \mathrm{C} \mathrm{NMR}\left(100 \mathrm{MHz}, \mathrm{CDCl}_{3}\right) \delta 144.00,139.47,132.47$, $129.89,127.71,127.63,126.72,126.41,121.38(\mathrm{~d}, J=255.7 \mathrm{~Hz}), 71.10(\mathrm{~d}, J=2.3$ Hz), 51.40, 50.13, 45.67, 39.07, 37.89, 27.47, 27.16, 26.75, 26.69, 26.15, 21.58. HRMS: m/z (ESI) calculated [M+NH$]^{+}:$499.2237, measured: 499.2241.<smiles>FC(F)(F)OC1CN([125I])CC2(CCCCC2)C1</smiles> 
The title compound was obtained as white solid. (47 mg, 60\%). ${ }^{1} \mathrm{H}$ NMR (400 MHz, $\left.\mathrm{CDCl}_{3}\right) \delta 7.64(\mathrm{~d}, J=8.3 \mathrm{~Hz}, 2 \mathrm{H}), 7.34(\mathrm{~d}, J=8.0 \mathrm{~Hz}, 2 \mathrm{H}), 4.40-4.50(\mathrm{~m}, 1 \mathrm{H}), 3.94$ (dd, $J=11.0,5.0 \mathrm{~Hz}, 1 \mathrm{H}), 3.57(\mathrm{~d}, J=11.7 \mathrm{~Hz}, 1 \mathrm{H}), 2.44$ (s, 3H), 2.25 (t, $J=10.4 \mathrm{~Hz}$, 1H), $2.04-1.92(\mathrm{~m}, 2 \mathrm{H}), 1.60-1.70(\mathrm{~m}, 1 \mathrm{H}), 1.55-1.43(\mathrm{~m}, 4 \mathrm{H}), 1.43-1.33(\mathrm{~m}, 3 \mathrm{H})$, $1.32-1.22(\mathrm{~m}, 2 \mathrm{H}), 1.12(\mathrm{dd}, J=13.0,10.9 \mathrm{~Hz}, 1 \mathrm{H}) .{ }^{19} \mathrm{~F} \mathrm{NMR}\left(376 \mathrm{MHz}, \mathrm{CDCl}_{3}\right) \delta$ -58.41 (s). ${ }^{13} \mathrm{C}$ NMR (100 MHz, $\left.\mathrm{CDCl}_{3}\right) \delta 143.78,133.34,129.81,127.44,121.41$ (q, $J=255.7 \mathrm{~Hz}), 70.76(\mathrm{q}, J=2.3 \mathrm{~Hz}), 53.73,50.16,41.58,37.03,34.70,32.43,26.11$, 21.53, 21.36, 21.10. HRMS: m/z (ESI-TOF) calculated $[\mathrm{M}+\mathrm{H}]^{+}:$392.1502, measured: 392.1505 .

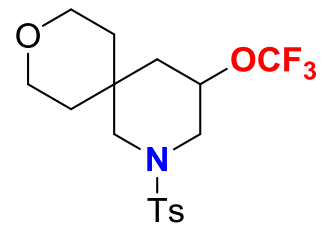

20

The title compound was obtained as white solid. (55 mg, 71\%). ${ }^{1} \mathrm{H}$ NMR (400 MHz, $\left.\mathrm{CDCl}_{3}\right) \delta 7.65(\mathrm{~d}, J=8.2 \mathrm{~Hz}, 2 \mathrm{H}), 7.34(\mathrm{~d}, J=8.1 \mathrm{~Hz}, 2 \mathrm{H}), 4.40-4.50(\mathrm{~m}, 1 \mathrm{H}), 3.87$ $(\mathrm{dd}, J=11.2,4.6 \mathrm{~Hz}, 1 \mathrm{H}), 3.74-3.53(\mathrm{~m}, 5 \mathrm{H}), 2.47-2.38(\mathrm{~m}, 4 \mathrm{H}), 2.21$ (d, $J=11.9$ $\mathrm{Hz}, 1 \mathrm{H}), 1.99$ (dd, $J=13.3,4.0 \mathrm{~Hz}, 1 \mathrm{H}), 1.78-1.69(\mathrm{~m}, 1 \mathrm{H}), 1.62-1.47$ (m, 2H), $1.40(\mathrm{dd}, J=10.4,3.5 \mathrm{~Hz}, 1 \mathrm{H}), 1.28(\mathrm{dd}, J=13.2,10.4 \mathrm{~Hz}, 1 \mathrm{H}) .{ }^{19} \mathrm{~F}$ NMR $(376 \mathrm{MHz}$, $\left.\mathrm{CDCl}_{3}\right) \delta-58.52$ (s). ${ }^{13} \mathrm{C}$ NMR (100 MHz, $\left.\mathrm{CDCl}_{3}\right) \delta 144.03,133.31,129.90,127.42$, 121.37 (q, $J=255.9 \mathrm{~Hz}), 70.26(\mathrm{q}, J=2.4 \mathrm{~Hz}), 63.21,63.04,52.83,50.04,41.56$, 36.16, 32.90, 32.58, 21.54. HRMS: m/z (ESI-TOF) calculated $[\mathrm{M}+\mathrm{H}]^{+}:$394.1294, measured: 394.1297.

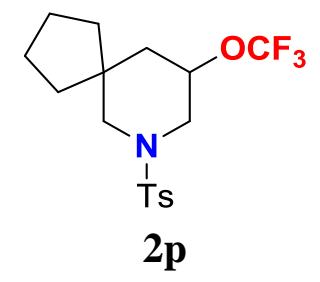

The title compound was obtained as white solid. (51 mg, 68\%). ${ }^{1} \mathrm{H}$ NMR (400 MHz, $\left.\mathrm{CDCl}_{3}\right) \delta 7.63(\mathrm{~d}, J=8.2 \mathrm{~Hz}, 2 \mathrm{H}), 7.33(\mathrm{~d}, J=8.2 \mathrm{~Hz}, 2 \mathrm{H}), 4.30-4.40(\mathrm{~m}, 1 \mathrm{H}), 3.90$ $(\mathrm{dd}, J=11.0,4.8 \mathrm{~Hz}, 1 \mathrm{H}), 3.26(\mathrm{~d}, J=11.4 \mathrm{~Hz}, 1 \mathrm{H}), 2.43(\mathrm{~s}, 3 \mathrm{H}), 2.27$ (t, $J=10.3 \mathrm{~Hz}$, 
1H), $2.08(\mathrm{~d}, J=11.4 \mathrm{~Hz}, 1 \mathrm{H}), 1.88(\mathrm{dd}, J=13.1,4.5 \mathrm{~Hz}, 1 \mathrm{H}), 1.82(\mathrm{dd}, J=12.9,6.3$ $\mathrm{Hz}, 1 \mathrm{H}), 1.74-1.56(\mathrm{~m}, 4 \mathrm{H}), 1.48-1.29$ (m, 4H). ${ }^{19} \mathrm{~F}$ NMR $\left(376 \mathrm{MHz}, \mathrm{CDCl}_{3}\right) \delta$ -58.35 (s). ${ }^{13} \mathrm{C}$ NMR (100 MHz, $\left.\mathrm{CDCl}_{3}\right) \delta 143.83,133.21,129.80,127.48,121.42$ (q, $J=255.7 \mathrm{~Hz}), 71.94(\mathrm{q}, J=2.3 \mathrm{~Hz}), 54.44,49.68,42.90,42.42,37.91,35.14,24.76$, 24.02, 21.51. HRMS: m/z (ESI-TOF) calculated $[\mathrm{M}+\mathrm{H}]^{+}:$378.1345, measured: 378.1350 .
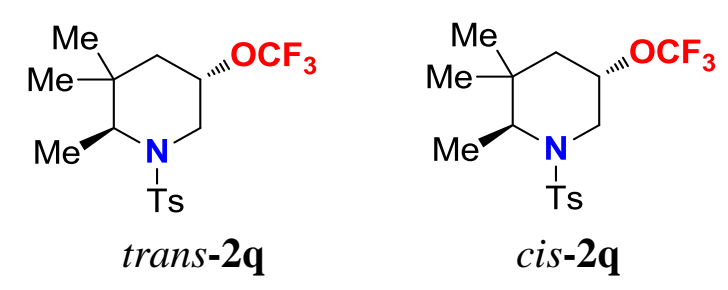

The title compound was obtained as light-yellow oil $(60 \mathrm{mg}, 80 \%$ yield, trans:cis $=$ 1.6:1). cis-2q: ${ }^{1} \mathrm{H}$ NMR $\left(400 \mathrm{MHz}, \mathrm{CDCl}_{3}\right) \delta 7.67(\mathrm{~d}, J=8.3 \mathrm{~Hz}, 2 \mathrm{H}), 7.30(\mathrm{~d}, J=8.1$ $\mathrm{Hz}, 2 \mathrm{H}), 4.30-4.41(\mathrm{~m}, 1 \mathrm{H}), 3.99(\mathrm{dd}, J=12.3,5.6 \mathrm{~Hz}, 1 \mathrm{H}), 3.73$ (q, $J=7.0 \mathrm{~Hz}, 1 \mathrm{H})$, $2.72(\mathrm{dd}, J=11.9,11.2 \mathrm{~Hz}, 1 \mathrm{H}), 2.42$ (s, 3H), $1.70(\mathrm{dd}, J=13.3,5.1 \mathrm{~Hz}, 1 \mathrm{H}), 1.55$ (t, $J=12.7 \mathrm{~Hz}, 1 \mathrm{H}), 1.10$ (s, 3H), 0.89 (s, 3H), 0.88 (s, 3H). ${ }^{19} \mathrm{~F} \mathrm{NMR}\left(376 \mathrm{MHz}, \mathrm{CDCl}_{3}\right)$ $\delta-58.21$ (s, 3F). ${ }^{13} \mathrm{C}$ NMR (100 MHz, $\left.\mathrm{CDCl}_{3}\right) \delta 143.37,137.59,129.79,126.84$, $121.41(\mathrm{q}, J=255.7 \mathrm{~Hz}), 71.17(\mathrm{q}, J=2.2 \mathrm{~Hz}), 56.54,42.55,38.19,35.31,27.45$, 26.75, 21.50, 11.22. trans-2q: ${ }^{1} \mathrm{H}$ NMR (400 MHz, $\left.\mathrm{CDCl}_{3}\right) \delta 7.70(\mathrm{~d}, J=8.0 \mathrm{~Hz}, 2 \mathrm{H})$, $7.27(\mathrm{~d}, J=8.6 \mathrm{~Hz}, 2 \mathrm{H}), 4.31-4.39(\mathrm{~m}, 1 \mathrm{H}), 3.89(\mathrm{~d}, J=14.7 \mathrm{~Hz}, 1 \mathrm{H}), 3.74(\mathrm{q}, J=6.7$ $\mathrm{Hz}, 1 \mathrm{H}), 3.14$ (d, $J=14.4 \mathrm{~Hz}, 1 \mathrm{H}), 2.41(\mathrm{~s}, 3 \mathrm{H}), 1.62$ (d, $J=12.0 \mathrm{~Hz}, 2 \mathrm{H}), 1.04$ (d, $J$ $=6.8 \mathrm{~Hz}, 3 \mathrm{H}), 0.97(\mathrm{~s}, 3 \mathrm{H}), 0.83(\mathrm{~s}, 3 \mathrm{H}) .{ }^{19} \mathrm{~F} \mathrm{NMR}\left(376 \mathrm{MHz}, \mathrm{CDCl}_{3}\right) \delta-58.89(\mathrm{~s}$, $3 \mathrm{~F}) .{ }^{13} \mathrm{C} \mathrm{NMR}\left(100 \mathrm{MHz}, \mathrm{CDCl}_{3}\right) \delta 143.20,137.41,129.43,127.27,121.44$ (q, $J=$ $254.9 \mathrm{~Hz}$ ), 71.77 (q, $J=2.4 \mathrm{~Hz}$ ), 57.19, 42.93, 36.02, 33.09, 28.26, 28.01, 21.49, 12.23. HRMS: m/z (EI) calculated [M]: 365.1273, measured: 365.1270.

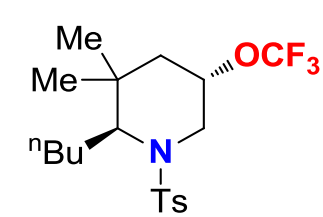

trans-2r

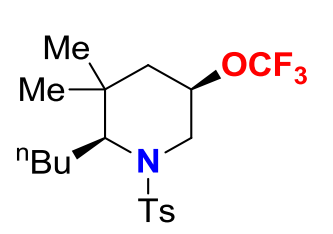

cis-2r

The title compound was obtained as white solid $(59 \mathrm{mg}, 72 \%$ yield, trans: $\mathrm{cis}=$ 1.6:1). cis-2r: ${ }^{1} \mathrm{H}$ NMR (400 MHz, $\left.\mathrm{CDCl}_{3}\right) \delta 7.69(\mathrm{~d}, J=8.3 \mathrm{~Hz}, 2 \mathrm{H}), 7.28(\mathrm{~d}, J=8.1$ $\mathrm{Hz}, 2 \mathrm{H}), 4.24-4.35$ (m, 1H), 3.90 (dd, $J=13.2,5.5 \mathrm{~Hz}, 1 \mathrm{H}), 3.62$ (dd, $J=9.2,5.3$ 
$\mathrm{Hz}, 1 \mathrm{H}), 2.66(\mathrm{dd}, J=13.2,10.9 \mathrm{~Hz}, 1 \mathrm{H}), 2.42(\mathrm{~s}, 3 \mathrm{H}), 1.68(\mathrm{dd}, J=13.0,5.0 \mathrm{~Hz}$, $1 \mathrm{H}), 1.64-1.54(\mathrm{~m}, 1 \mathrm{H}), 1.50-1.08(\mathrm{~m}, 6 \mathrm{H}), 1.06(\mathrm{~s}, 3 \mathrm{H}), 0.93(\mathrm{~s}, 3 \mathrm{H}), 0.78(\mathrm{t}, J=$ $7.1 \mathrm{~Hz}, 3 \mathrm{H}) .{ }^{19} \mathrm{~F}$ NMR $\left(376 \mathrm{MHz}, \mathrm{CDCl}_{3}\right) \delta-58.19(\mathrm{~s}, 3 \mathrm{~F}) .{ }^{13} \mathrm{C} \mathrm{NMR}(100 \mathrm{MHz}$, $\left.\mathrm{CDCl}_{3}\right) \delta 143.24,137.95,129.53,126.99,121.42(\mathrm{q}, J=255.6 \mathrm{~Hz}), 70.85$ (q, $J=1.9$ $\mathrm{Hz}), 61.66,42.93,39.00,35.85$, 29.68, 27.76, 27.45, 26.31, 22.64, 21.45, 13.85. trans-2r: ${ }^{1} \mathrm{H}$ NMR $\left(400 \mathrm{MHz}, \mathrm{CDCl}_{3}\right) \delta 7.70(\mathrm{~d}, J=8.1 \mathrm{~Hz}, 2 \mathrm{H}), 7.25(\mathrm{~d}, J=8.5 \mathrm{~Hz}$, $2 \mathrm{H}), 4.23-4.28(\mathrm{~m}, 1 \mathrm{H}), 3.89(\mathrm{~d}, J=15.2 \mathrm{~Hz}, 1 \mathrm{H}), 3.68(\mathrm{t}, J=6.4 \mathrm{~Hz}, 1 \mathrm{H}), 3.06(\mathrm{~d}$, $J=15.0 \mathrm{~Hz}, 1 \mathrm{H}), 2.39(\mathrm{~s}, 3 \mathrm{H}), 1.64(\mathrm{dd}, J=15.4,3.5 \mathrm{~Hz}, 1 \mathrm{H}), 1.54(\mathrm{~d}, J=15.2 \mathrm{~Hz}$, 1H), 1.50-1.20 (m, 6H), $0.90(\mathrm{~s}, 3 \mathrm{H}), 0.88(\mathrm{t}, J=7.0 \mathrm{~Hz}, 3 \mathrm{H}), 0.85(\mathrm{~s}, 3 \mathrm{H}) .{ }^{19} \mathrm{~F}$ NMR $\left(376 \mathrm{MHz}, \mathrm{CDCl}_{3}\right) \delta-59.07$ (s, 3F). ${ }^{13} \mathrm{C} \mathrm{NMR}\left(100 \mathrm{MHz}, \mathrm{CDCl}_{3}\right) \delta 142.97,137.97$, $129.16127 .38,121.32(\mathrm{q}, J=254.7 \mathrm{~Hz}), 71.90(\mathrm{q}, J=2.4 \mathrm{~Hz}), 62.33,43.27,36.32$, $33.39,29.32,28.91,28.38,26.52,22.78,21.41,13.91$. HRMS: m/z (EI) calculated [M]: 407.1742, measured: 407.1746. Structure of trans-2r was defined by x-ray.

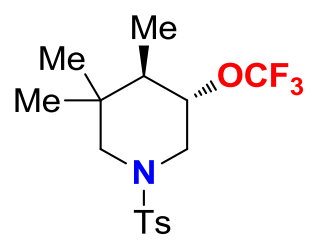

trans-2s

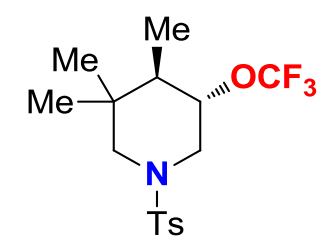

cis-2s

The reaction provided the mixture of isomer with trans:cis $=5.3: 1$. trans-2s isomer was isolated as white solid (39 mg, 53\% yield, ): ${ }^{1} \mathrm{H}$ NMR (400 $\mathrm{MHz}, \mathrm{CDCl}_{3}$ ) $\delta 7.63(\mathrm{~d}, J=8.2 \mathrm{~Hz}, 2 \mathrm{H}), 7.34(\mathrm{~d}, J=8.1 \mathrm{~Hz}, 2 \mathrm{H}), 4.12-4.00(\mathrm{~m}, 2 \mathrm{H}), 3.35(\mathrm{dd}, J=$ 11.5, $1.7 \mathrm{~Hz}, 1 \mathrm{H}), 2.44$ (s, 3H), $2.18-2.09$ (m, 1H), 1.97 (d, J=11.5 Hz, 1H), $1.25-$ $1.15(\mathrm{~m}, 1 \mathrm{H}), 0.98(\mathrm{~s}, 3 \mathrm{H}), 0.96(\mathrm{~d}, J=6.7 \mathrm{~Hz}, 3 \mathrm{H}), 0.91(\mathrm{~s}, 3 \mathrm{H}) .{ }^{19} \mathrm{~F}$ NMR $(376 \mathrm{MHz}$, $\left.\mathrm{CDCl}_{3}\right) \delta-58.15(\mathrm{~s}, 3 \mathrm{~F}) .{ }^{13} \mathrm{C} \mathrm{NMR}\left(100 \mathrm{MHz}, \mathrm{cdcl}_{3}\right) \delta 143.86,132.96,129.82,127.52$, $121.57(\mathrm{q}, J=255.5 \mathrm{~Hz}), 76.36(\mathrm{q}, J=1.7 \mathrm{~Hz}), 58.17,50.30,44.36,34.89,26.24$, 21.54, 19.05, 10.64. HRMS: m/z (EI) calculated [M]: 365.1273, measured: 365.1270.

cis-2s was not isolated as purity product; the yield was calculated to be $9 \%$ according to crude ${ }^{1} \mathrm{H}$ NMR. 


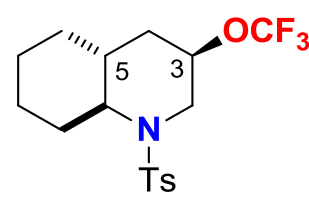

3,5-trans-2t

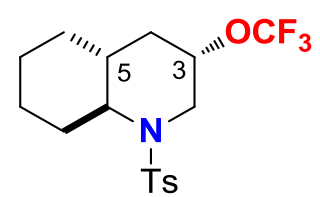

3,5-cis-3t

The title compound was obtained as white solid $(57 \mathrm{mg}, 76 \%$ yield, 3,5-trans:3.5-cis $=1.36: 1) .3,5$-cis-2t: ${ }^{1} \mathrm{H}$ NMR $\left(400 \mathrm{MHz}, \mathrm{CDCl}_{3}\right) \delta 7.66(\mathrm{~d}, J=8.3$ $\mathrm{Hz}, 2 \mathrm{H}), 7.30(\mathrm{~d}, J=8.0 \mathrm{~Hz}, 2 \mathrm{H}), 4.42-4.30(\mathrm{~m}, 2 \mathrm{H}), 2.77-2.68(\mathrm{~m}, 1 \mathrm{H}), 2.43(\mathrm{~s}$, $3 \mathrm{H}), 2.38(\mathrm{dd}, J=10.9,2.9 \mathrm{~Hz}, 1 \mathrm{H}), 2.17(\mathrm{dd}, J=13.1,3.3 \mathrm{~Hz}, 1 \mathrm{H}), 2.10(\mathrm{~d}, J=12.2$ $\mathrm{Hz}, 1 \mathrm{H}), 1.87-1.73(\mathrm{~m}, 1 \mathrm{H}), 1.73-1.60(\mathrm{~m}, 2 \mathrm{H}), 1.58-1.47(\mathrm{~m}, 1 \mathrm{H}), 1.27-0.94$ $(\mathrm{m}, 5 \mathrm{H}) .{ }^{19} \mathrm{~F}$ NMR $\left(376 \mathrm{MHz}, \mathrm{CDCl}_{3}\right) \delta-58.17(\mathrm{~s}, 3 \mathrm{~F}) .{ }^{13} \mathrm{C} \mathrm{NMR}\left(100 \mathrm{MHz}, \mathrm{CDCl}_{3}\right) \delta$ 143.42, 137.23, 129.68, 127.10, 121.41 (q, $J=255.6 \mathrm{~Hz}), 72.82$ (q, $J=2.2 \mathrm{~Hz}), 64.44$, 52.04, 39.58, 38.41, 32.92, 30.74, 25.71, 24.94, 21.50. 3, 5-trans-2t: ${ }^{1} \mathrm{H}$ NMR (400 $\left.\mathrm{MHz}, \mathrm{CDCl}_{3}\right) \delta 7.71(\mathrm{~d}, J=8.3 \mathrm{~Hz}, 2 \mathrm{H}), 7.30(\mathrm{~d}, J=8.0 \mathrm{~Hz}, 2 \mathrm{H}), 4.36-4.43(\mathrm{~m}, 1 \mathrm{H})$, $3.84(\mathrm{dd}, J=14.3,6.2 \mathrm{~Hz}, 1 \mathrm{H}), 3.39(\mathrm{dd}, J=14.3,4.4 \mathrm{~Hz}, 1 \mathrm{H}), 2.81(\mathrm{td}, J=10.8,3.4$ $\mathrm{Hz}, 1 \mathrm{H}), 2.43(\mathrm{~s}, 3 \mathrm{H}), 2.26(\mathrm{dd}, J=11.8,2.4 \mathrm{~Hz}, 1 \mathrm{H}), 1.84-1.62(\mathrm{~m}, 5 \mathrm{H}), 1.42-$ $1.29(\mathrm{~m}, 1 \mathrm{H}), 1.29-1.16(\mathrm{~m}, 3 \mathrm{H}), 1.07-0.91(\mathrm{~m}, 1 \mathrm{H}) .{ }^{19} \mathrm{~F}$ NMR $\left(376 \mathrm{MHz}, \mathrm{CDCl}_{3}\right)$ $\delta-58.45$ (s, 3F). ${ }^{13} \mathrm{C}$ NMR (100 MHz, $\left.\mathrm{CDCl}_{3}\right) \delta$ 143.40, 137.98, 129.70, 127.03, $121.55(\mathrm{q}, J=255.2 \mathrm{~Hz}), 71.48(\mathrm{q}, J=2.2 \mathrm{~Hz}), 63.18,47.18,35.65,35.48,32.50$, 31.78, 25.52, 25.30, 21.50. HRMS: m/z (EI) calculated [M]: 377.1273, measured: 377.1269 .

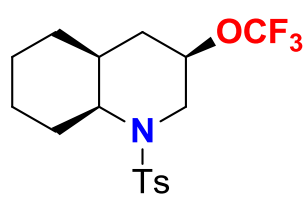

cis-2u<smiles>[13CH3]N1C[C@@H](OC(F)(F)F)C[C@@H]2CCCC[C@H]21</smiles>

trans-2u

The title compound was obtained as light-yellow oil (58 mg, $77 \%$ yield, 3,5-cis-2u :3,5-trans-2u = 3.6:1). 3,5-cis-2u: ${ }^{1} \mathrm{H}$ NMR (400 MHz, $\left.\mathrm{CDCl}_{3}\right) \delta 7.69$ (d, $J$ $=8.2 \mathrm{~Hz}, 2 \mathrm{H}), 7.30(\mathrm{~d}, J=8.1 \mathrm{~Hz}, 2 \mathrm{H}), 4.11-3.87(\mathrm{~m}, 3 \mathrm{H}), 2.87(\mathrm{dd}, J=12.4,10.3$ $\mathrm{Hz}, 1 \mathrm{H}), 2.43$ (s, 3H), $1.92-1.60(\mathrm{~m}, 6 \mathrm{H}), 1.56-1.14(\mathrm{~m}, 5 \mathrm{H}) .{ }^{19} \mathrm{~F}$ NMR $(376 \mathrm{MHz}$, $\left.\mathrm{CDCl}_{3}\right) \delta-58.25(\mathrm{~s}, 3 \mathrm{~F}) .{ }^{13} \mathrm{C} \mathrm{NMR}\left(100 \mathrm{MHz}, \mathrm{CDCl}_{3}\right) \delta 143.43,138.00,129.85$, $126.73,121.36(\mathrm{q}, J=255.4 \mathrm{~Hz}), 73.04(\mathrm{q}, J=2.3 \mathrm{~Hz}), 53.91,43.13,33.67,30.57$, 30.42, 25.28, 23.30, 21.51, 19.61. 3,5-trans-2u : ${ }^{1} \mathrm{H}$ NMR (400 MHz, $\left.\mathrm{CDCl}_{3}\right) \delta 7.73$ 
(d, $J=8.3 \mathrm{~Hz}, 2 \mathrm{H}), 7.26(\mathrm{~d}, J=7.9 \mathrm{~Hz}, 2 \mathrm{H}), 4.40(\mathrm{~s}, 1 \mathrm{H}), 3.97(\mathrm{~d}, J=15.0 \mathrm{~Hz}, 1 \mathrm{H})$, $3.93-3.85(\mathrm{~m}, 1 \mathrm{H}), 3.17$ (d, $J=14.8 \mathrm{~Hz}, 1 \mathrm{H}), 2.41(\mathrm{~s}, 3 \mathrm{H}), 2.13-1.59(\mathrm{~m}, 6 \mathrm{H}), 1.56$ $-1.11(\mathrm{~m}, 5 \mathrm{H}) .{ }^{19} \mathrm{~F}$ NMR $\left(376 \mathrm{MHz}, \mathrm{CDCl}_{3}\right) \delta-58.71(\mathrm{~s}, 3 \mathrm{~F}) .{ }^{13} \mathrm{C} \mathrm{NMR}(100 \mathrm{MHz}$, $\left.\mathrm{CDCl}_{3}\right) \delta 143.01,137.74,129.37,127.18,121.46(\mathrm{q}, J=256.0 \mathrm{~Hz}), 71.95(\mathrm{q}, J=1.9$ Hz), 54.38, 43.44, 30.38, 28.27 27.91, 25.18, 23.72, 21.48, 19.46. HRMS: m/z (EI) calculated [M]: 377.1273, measured: 377.1266. 


\section{Crystal Structure of 2f, $2 r$ and 10.}

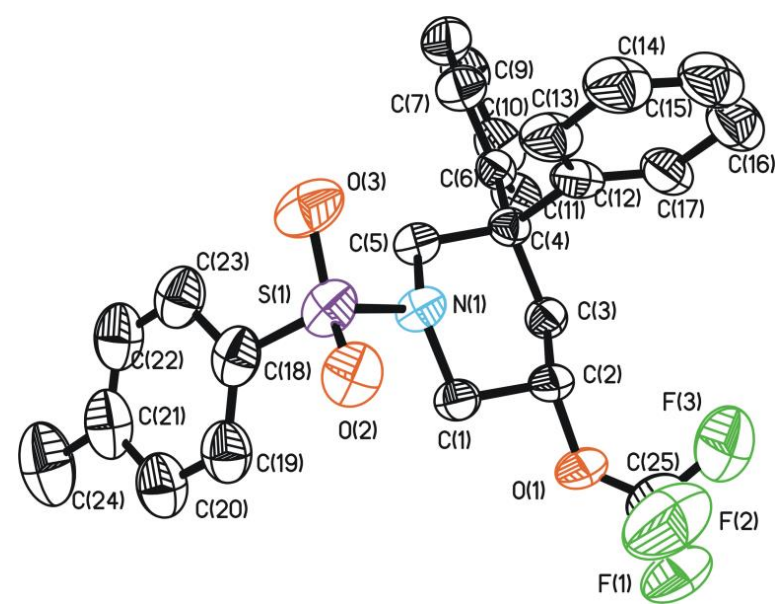

X-ray structure of $\mathbf{2 f}$

Table S1. Crystal data and structure refinement for $\mathbf{2 f}$.

Identification code

$\operatorname{dm} 15470$

Empirical formula

C25 H24 F3 N O3 S

Formula weight

475.51

Temperature

$296.15 \mathrm{~K}$

Wavelength

$0.71073 \AA$

Crystal system

Monoclinic

Space group

P 1 21/c 1

Unit cell dimensions

$$
\begin{array}{ll}
\mathrm{a}=9.9716(14) \AA & \mathrm{a}=90^{\circ} . \\
\mathrm{b}=25.875(3) \AA & \mathrm{b}=
\end{array}
$$

$102.985(3)^{\circ}$.

$$
\mathrm{c}=9.5523(13) \AA \quad \mathrm{g}=90^{\circ} .
$$

Volume

2401.6(6) $\AA^{3}$

Z

4

Density (calculated)

$1.315 \mathrm{Mg} / \mathrm{m}^{3}$ 
Absorption coefficient

$\mathrm{F}(000)$

Crystal size

Theta range for data collection

Index ranges

Reflections collected

Independent reflections

Completeness to theta $=26.000^{\circ}$

Absorption correction

Max. and min. transmission

Refinement method

Data / restraints / parameters

Goodness-of-fit on $\mathrm{F}^{2}$

Final R indices [I $>2 \operatorname{sigma}(\mathrm{I})]$

$\mathrm{R}$ indices (all data)

Extinction coefficient

Largest diff. peak and hole
$0.184 \mathrm{~mm}^{-1}$

992

$0.2 \times 0.15 \times 0.08 \mathrm{~mm}^{3}$

1.574 to $25.999^{\circ}$.

$-12<=\mathrm{h}<=12,-30<=\mathrm{k}<=31,-11<=\mathrm{l}<=7$

17501

$4704[\mathrm{R}$ (int) $=0.0673]$

$99.9 \%$

Semi-empirical from equivalents

0.7456 and 0.6682

Full-matrix least-squares on $\mathrm{F}^{2}$

4704 / 171 / 299

1.023

$\mathrm{R} 1=0.0831, \mathrm{wR} 2=0.2229$

$\mathrm{R} 1=0.1670, \mathrm{wR} 2=0.2876$

$\mathrm{n} / \mathrm{a}$

0.799 and -0.580 e. $\AA^{-3}$ 


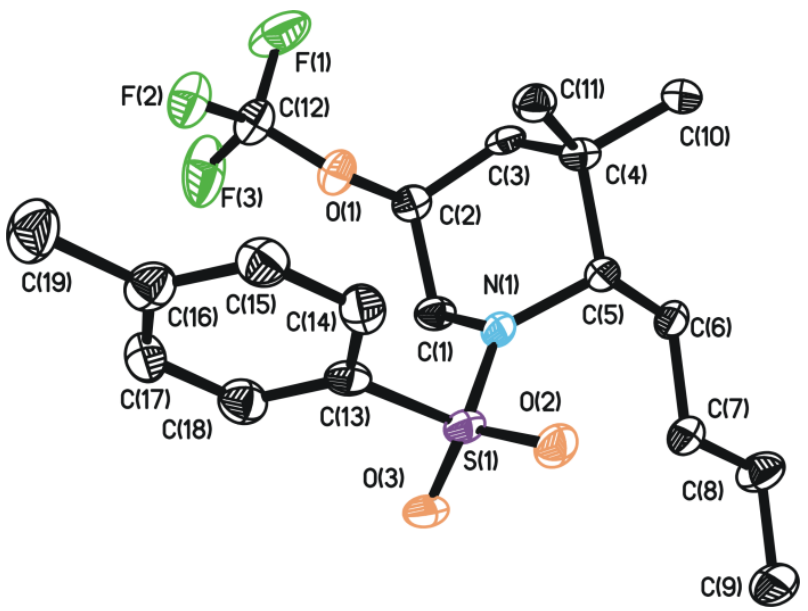

X-ray structure of $\mathbf{2 r}$

Table S2. Crystal data and structure refinement for $\mathbf{2 r}$.

Identification code

Empirical formula

Formula weight

Temperature

Wavelength

Crystal system

Space group

Unit cell dimensions

Volume

Z

Density (calculated)

Absorption coefficient

$\mathrm{F}(000)$ mo_dm15698_0m

\section{C19 H28 F3 N O3 S}

407.48

$130 \mathrm{~K}$

$0.71073 \AA$

Triclinic

P -1

$$
\begin{array}{ll}
\mathrm{a}=8.376(4) \AA & \alpha=86.431(8)^{\circ} . \\
\mathrm{b}=12.224(6) \AA & \beta=89.627(8)^{\circ} . \\
\mathrm{c}=21.474(10) \AA & \gamma=71.394(7)^{\circ} .
\end{array}
$$

2079.5(16) $\AA^{3}$

4

$1.302 \mathrm{Mg} / \mathrm{m}^{3}$

$0.200 \mathrm{~mm}^{-1}$

864 
Crystal size

Theta range for data collection

Index ranges

Reflections collected

Independent reflections

Completeness to theta $=26.000^{\circ}$

Absorption correction

Max. and min. transmission

Refinement method

Data / restraints / parameters

Goodness-of-fit on $\mathrm{F}^{2}$

Final R indices [I $>2 \operatorname{sigma}(\mathrm{I})]$

$\mathrm{R}$ indices (all data)

Extinction coefficient

Largest diff. peak and hole e. $\AA^{-3 \mathrm{v}}$
$0.15 \times 0.1 \times 0.05 \mathrm{~mm}^{3}$

1.761 to $27.653^{\circ}$.

$-10<=\mathrm{h}<=10,-15<=\mathrm{k}<=15,0<=\mathrm{l}<=27$

9460

$9460[\mathrm{R}($ int $)=?]$

$99.5 \%$

Semi-empirical from equivalents

0.7455 and 0.4974

Full-matrix least-squares on $\mathrm{F}^{2}$

9460 / 0 / 496

0.885

$\mathrm{R} 1=0.0605, \mathrm{wR} 2=0.1005$

$\mathrm{R} 1=0.1490, \mathrm{wR} 2=0.1223$

$\mathrm{n} / \mathrm{a}$

0.368 and -0.468 


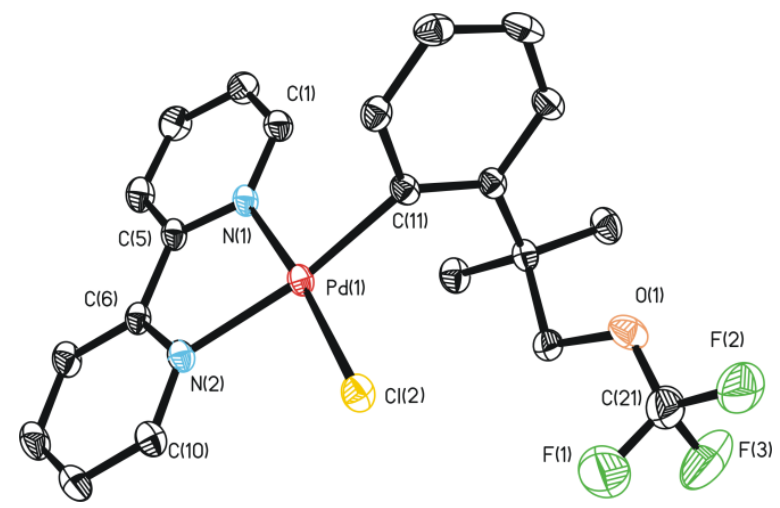

X-ray structure of complex $\mathbf{1 0}$

Table S3. Crystal data and structure refinement for $\mathbf{1 0 .}$

Identification code

Empirical formula

Formula weight

Temperature

Wavelength

Crystal system

Space group

Unit cell dimensions

Volume

Z

Density (calculated)

Absorption coefficient

$\mathrm{F}(000)$

Crystal size mo_dm15767_0m

C21 H20 Cl F3 N2 O Pd

515.24

$130 \mathrm{~K}$

$0.71073 \AA$

Triclinic

$\mathrm{P}-1$

$a=9.1462(11) \AA$

$\alpha=76.519(2)^{\circ}$.

$\mathrm{b}=9.9402(12) \AA$

$\beta=77.210(2)^{\circ}$.

$c=13.4540(17) \AA$ $\gamma=78.063(2)^{\circ}$.
1144.3(2) $\AA^{3}$

2

$1.495 \mathrm{Mg} / \mathrm{m}^{3}$

$0.964 \mathrm{~mm}^{-1}$

516

$0.12 \times 0.05 \times 0.03 \mathrm{~mm}^{3}$ 
Theta range for data collection

Index ranges

Reflections collected

Independent reflections

Completeness to theta $=26.000^{\circ}$

Absorption correction

Max. and min. transmission

Refinement method

Data / restraints / parameters

Goodness-of-fit on $\mathrm{F}^{2}$

Final $\mathrm{R}$ indices [I $>2 \operatorname{sigma}(\mathrm{I})]$

$\mathrm{R}$ indices (all data)

Extinction coefficient

Largest diff. peak and hole
1.583 to $30.878^{\circ}$.

$-13<=\mathrm{h}<=12,-14<=\mathrm{k}<=14,-19<=\mathrm{l}<=17$

11894

$7120[\mathrm{R}($ int $)=0.0308]$

$99.7 \%$

Semi-empirical from equivalents

0.7461 and 0.6394

Full-matrix least-squares on $\mathrm{F}^{2}$

$7120 / 1 / 264$

0.956

$\mathrm{R} 1=0.0401, \mathrm{wR} 2=0.0840$

$\mathrm{R} 1=0.0560, \mathrm{wR} 2=0.0893$

$\mathrm{n} / \mathrm{a}$

0.708 and -0.924 e. $\AA^{-3}$

(S1) Zhang, C.; Vicic, D. A. Organometallics 2012, 31, 7812.

(S2) (a) Noftle, R.; Cady, G. H. Inorg. Chem. 1965, 4, 1010. (b) Huang, C.; Liang, T.; Harada, S.; Lee, E.; Ritter, T. J. Am. Chem. Soc. 2011, 133, 13308.

(S3) Wu, T.; Yin, G.; Liu, G. J. Am. Chem. Soc. 2009, 131, 16354.

(S4) White, P. B.; Stahl, S. S. J. Am. Chem. Soc. 2011, 133, 18594.

(S5) (a) Campora, J.; Lopez, J. A.; Palma, P.; Rio, D.; Carmona, E.; Valerga, P.; Graiff, C.; Tiripicchio, A. Inorg. Chem. 2001, 40, 4116. (b) Racowski, J. M.; Gary, J. B.; Sanford, M. S.. Angew. Chem. Int. Ed. 2012, 51, 3414. 